\title{
Consistency of the Duhem Model with Hysteresis
}

\author{
Mohammad Fuad Mohammad Naser and Fayçal Ikhouane \\ Universitat Politècnica de Catalunya, Escola Universitària d'Enginyeria Tècnica Industrial de Barcelona, \\ Departament de Matemàtica Aplicada III, Comte d'Urgell 187, 08036 Barcelona, Spain
}

Correspondence should be addressed to Fayçal Ikhouane; faycal.ikhouane@upc.edu

Received 26 March 2013; Accepted 27 July 2013

Academic Editor: Piermarco Cannarsa

Copyright (C) 2013 M. F. Mohammad Naser and F. Ikhouane. This is an open access article distributed under the Creative Commons Attribution License, which permits unrestricted use, distribution, and reproduction in any medium, provided the original work is properly cited.

\begin{abstract}
The Duhem model, widely used in structural, electrical, and mechanical engineering, gives an analytical description of a smooth hysteretic behavior. In practice, the Duhem model is mostly used within the following black-box approach: given a set of experimental input-output data, how to tune the model so that its output matches the experimental data. It may happen that a Duhem model presents a good match with the experimental real data for a specific input but does not necessarily keep significant physical properties which are inherent to the real data, independent of the exciting input. This paper presents a characterization of different classes of Duhem models in terms of their consistency with the hysteresis behavior.
\end{abstract}

\section{Introduction}

Hysteresis is a nonlinear behavior encountered in a wide variety of processes including biology, optics, electronics, ferroelectricity, magnetism, mechanics, structures, among other areas. The detailed modeling of hysteresis systems using the laws of Physics is an arduous task, and the obtained models are often too complex to be used in applications. For this reason, alternative models of these complex systems have been proposed [1-5]. These models do not come, in general, from the detailed analysis of the physical behavior of the systems with hysteresis. Instead, they combine some physical understanding of the system along with some kind of blackbox modeling.

One of the popular models for hysteresis is the Duhem model proposed in [6]. The generalized form of the Duhem model consists of an ordinary differential equation of the form $\dot{x}=f(x, u) g(\dot{u})$, where $u$ is the input and $x$ is the state or the output [7]. Other special forms of the model have been used, like the form $\dot{x}=f_{1}(x, u) \max \{\dot{u}, 0\}+f_{2}(x, u) \min \{\dot{u}, 0\}$ [8] or the semilinear form $\dot{x}=(A x+B u) g(\dot{u})$ [9]. Other important special cases of the Duhem model are the LuGre model of friction [10], the Dahl model of friction [11], and the Bouc-Wen model of hysteresis $[12,13]$. The Duhem model has been used to represent friction [7], electromagnetic behavior $[14,15]$, or hysteresis in magnetorheological dampers [16].
In the current literature, the Duhem model is mostly used within the following black-box approach: given a set of experimental input-output data, how to adjust the Duhem model so that the output of the model matches the experimental data? The use of system identification techniques is one practical way to perform this task. Once an identification method has been applied to tune the Duhem model, the resulting model is considered as a "good" approximation of the true hysteresis when the error between the experimental data and the output of the model is small enough. Then, this model is used to study the behavior of the true hysteresis under different excitations. By doing this, it is important to consider the following remark. It may happen that a Duhem model presents a good match with the experimental real data for a specific input but does not necessarily keep significant physical properties which are inherent to the real data, independent of the exciting input. In the current literature, this issue has been considered in $[17,18]$ regarding the passivity/dissipativity of Duhem model.

In this paper, we investigate the conditions under which the Duhem model is consistent with the hysteresis behavior. The concept of consistency is formalized in [19] where a general class of hysteresis operators is considered. The class of operators that are considered in [19] are the causal ones, with the additional condition that a constant input leads to 
a constant output. For these classes of systems, consistency has been defined formally. This property is useful in system modeling and identification as it limits the search for the system's parameters to those regions where consistency holds. From the results of [19], it can be concluded that to check consistency one has to consider the sequence of inputs $u_{\gamma}(t)=$ $u(t / \gamma), t \geq 0, \gamma>0$ and the corresponding sequence of outputs $x_{\gamma}$ with $\dot{x}_{\gamma}=f\left(x_{\gamma}, u_{\gamma}\right) g\left(\dot{u}_{\gamma}\right)$. For the Duhem model to represent a hysteresis system, it is necessary that the sequence of functions $t \rightarrow x_{\gamma}(\gamma t)$ converges uniformly when $\gamma \rightarrow \infty$. In this paper, we seek necessary conditions and sufficient ones for this uniform convergence to hold.

This paper is organized as follows. Section 2 presents the needed mathematical background. The problem statement is formalized in Section 3. A classification of functions $g$ that is used throughout the paper, is introduced in Section 4. Sections 5 and 6 present necessary conditions and sufficient ones for the Duhem model to be consistent with the hysteresis behavior. Conclusions are given in Section 7.

\section{Background Results}

This section summarizes the results obtained in [19].

A function $\nu: \mathbb{R} \rightarrow \mathbb{R}$ is said to be increasing (resp., decreasing) if $t_{1}<t_{2} \Rightarrow v\left(t_{1}\right)<v\left(t_{2}\right)$ (resp. $v\left(t_{1}\right)>v\left(t_{2}\right)$ ), and it is said to be nondecreasing (resp. nonincreasing) if $t_{1}<$ $t_{2} \Rightarrow v\left(t_{1}\right) \leq v\left(t_{2}\right)$ (resp., $v\left(t_{1}\right) \geq v\left(t_{2}\right)$ ).

The Lebesgue measure on $\mathbb{R}$ is denoted $\mu$. A subset of $\mathbb{R}$ is said to be measurable when it is Lebesgue measurable. Consider a function $p: I \subset \mathbb{R}_{+}=[0, \infty) \rightarrow \mathbb{R}^{m}$ for some interval $I$; the function $p$ is said to be measurable when $p$ is $(M, B)$-measurable where $B$ is the class of Borel sets of $\mathbb{R}^{m}$ and $M$ is the class of measurable sets of $\mathbb{R}_{+}$. For a measurable function $p: I \subset \mathbb{R}_{+} \rightarrow \mathbb{R}^{m},\|p\|_{\infty, I}$ denotes the essential supremum of the function $|p|$ on $I$ where $|\cdot|$ is the Euclidean norm on $\mathbb{R}^{m}$. When $I=\mathbb{R}_{+}$, and it is denoted simply $\|p\|_{\infty}$.

Consider the Sobolev space $W^{1, \infty}\left(\mathbb{R}_{+}, \mathbb{R}^{n}\right)$ of absolutely continuous functions $u: \mathbb{R}_{+} \rightarrow \mathbb{R}^{n}$, where $n$ is a positive integer. For this class of functions, the derivative $\dot{u}$ is defined almost every where with $\|u\|_{\infty}<\infty$ and $\|\dot{u}\|_{\infty}<$ $\infty$. Endowed with the norm $\|u\|_{1, \infty}=\max \left(\|u\|_{\infty},\|\dot{u}\|_{\infty}\right)$, $W^{1, \infty}\left(\mathbb{R}_{+}, \mathbb{R}^{n}\right)$ is a Banach space $[20]$.

For $u \in W^{1, \infty}\left(\mathbb{R}_{+}, \mathbb{R}^{n}\right)$, let $\rho_{u}: \mathbb{R}_{+} \rightarrow \mathbb{R}_{+}$be the total variation of $u$ on $[0, t]$; that is, $\rho_{u}(t)=\int_{0}^{t}|\dot{u}(\tau)| d \tau \in \mathbb{R}_{+}$. The function $\rho_{u}$ is well defined as $\dot{u} \in L_{\text {loc }}^{1}\left(\mathbb{R}_{+}, \mathbb{R}^{n}\right) .\left(L_{\text {loc }}^{1}\left(\mathbb{R}_{+}, \mathbb{R}^{n}\right)\right.$ is the space of locally integrable functions $\left.\mathbb{R}_{+} \rightarrow \mathbb{R}^{n}\right)$. It is nondecreasing and absolutely continuous. Denote $\rho_{u, \max }=$ $\lim _{t \rightarrow \infty} \rho_{u}(t)$. If $\rho_{u, \max }=\rho_{u}(t)$ for some $t \in \mathbb{R}_{+}$, let $I_{u}=$ $\left[0, \rho_{u, \max }\right]$ (in this case $\rho_{u, \max }$ is necessarily finite). On the other hand, if $\rho_{u, \max }>\rho_{u}(t)$ for all $t \in \mathbb{R}_{+}$, let $I_{u}=\left[0, \rho_{u, \max }\right)$ (in this case $\rho_{u, \max }$ may be finite or infinite).

Lemma 1. Let $u \in W^{1, \infty}\left(\mathbb{R}_{+}, \mathbb{R}^{n}\right)$ be nonconstant. Then, there exists a unique function $\psi_{u} \in W^{1, \infty}\left(I_{u}, \mathbb{R}^{n}\right)$ that satisfies $\psi_{u} \circ \rho_{u}=u$.

Consider the linear time scale change $s_{\gamma}(t)=t / \gamma$, for any $\gamma>0$ and $t \geq 0$. Let $\Xi$ be a set of initial conditions. Let $\mathscr{H}$ be an operator that maps the input function $u \in W^{1, \infty}\left(\mathbb{R}_{+}, \mathbb{R}^{n}\right)$ and initial condition $\xi^{0} \in \Xi$ to an output in $L^{\infty}\left(\mathbb{R}_{+}, \mathbb{R}^{m}\right)$, where $m$ is a positive integer. That is $\mathscr{H}: W^{1, \infty}\left(\mathbb{R}_{+}, \mathbb{R}^{n}\right) \times \Xi \rightarrow$ $L^{\infty}\left(\mathbb{R}_{+}, \mathbb{R}^{m}\right)$. We consider causal operators such that for all $\left(u_{1}, \xi^{0}\right),\left(u_{2}, \xi^{0}\right) \in W^{1, \infty}\left(\mathbb{R}_{+}, \mathbb{R}^{n}\right) \times \Xi$, if $u_{1}=u_{2}$ in $[0, \tau]$, then $\mathscr{H}\left(u_{1}, \xi^{0}\right)=\mathscr{H}\left(u_{2}, \xi^{0}\right)$ in [0, ] ], [1, page 60].

Let $\left(u, \xi^{0}\right) \in W^{1, \infty}\left(\mathbb{R}_{+}, \mathbb{R}^{n}\right) \times \Xi$ and let $y=\mathscr{H}\left(u, \xi^{0}\right) \in$ $L^{\infty}\left(\mathbb{R}_{+}, \mathbb{R}^{m}\right)$. In the rest of this work, only causal operators are considered. Additionally, we consider that the following holds.

Assumption 2. Let $\left(u, \xi^{0}\right) \in W^{1, \infty}\left(\mathbb{R}_{+}, \mathbb{R}^{n}\right) \times \Xi$ and $y=$ $\mathscr{H}\left(u, \xi^{0}\right) \in L^{\infty}\left(\mathbb{R}_{+}, \mathbb{R}^{m}\right)$; if $\exists \theta \in \mathbb{R}_{+}$such that $u$ is constant on $[\theta, \infty)$; then $y$ is constant on $[\theta, \infty)$.

Lemma 3. There exists a unique function $\varphi_{u} \in L^{\infty}\left(I_{u}, \mathbb{R}^{m}\right)$ that satisfies $\varphi_{u} \circ \rho_{u}=y$. Moreover, one has $\left\|\varphi_{u}\right\|_{\infty, I_{u}} \leq\|y\|_{\infty}$. If $y$ is continuous on $\mathbb{R}_{+}$, then $\varphi_{u}$ is continuous on $I_{u}$ and one has $\left\|\varphi_{u}\right\|_{\infty, I_{u}}=\|y\|_{\infty}$.

Definition 4. Let $u \in W^{1, \infty}\left(\mathbb{R}_{+}, \mathbb{R}^{n}\right)$ and initial condition $\xi^{0} \in \Xi$ be given. The operator $\mathscr{H}$ is said to be consistent with respect to input $u$ and initial condition $x_{0}$ if and only if the sequence of functions $\left\{\varphi_{u \circ s_{\gamma}}\right\}_{\gamma>0}$ converges in $L^{\infty}\left(I_{u}, \mathbb{R}^{m}\right)$ as $\gamma \rightarrow \infty$.

It is shown in [19] that for hysteresis process, the sequence of functions $\left\{\varphi_{u \circ s_{\gamma}}\right\}_{\gamma>0}$ converges in $L^{\infty}\left(I_{u}, \mathbb{R}^{m}\right)$ as $\gamma \rightarrow \infty$. This fact shows that consistency is a mathematical property that any model of hysteresis should satisfy.

\section{Problem Statement}

The generalized Duhem model is defined for almost all $t \geq 0$ by [7]

$$
\begin{gathered}
\dot{x}(t)=f(x(t), u(t)) g(\dot{u}(t)), \\
x(0)=x_{0},
\end{gathered}
$$

where $x_{0}$ and state $x(t)$ take values in $\mathbb{R}^{m}$ for some positive integer $m$, input $u \in W^{1, \infty}\left(\mathbb{R}_{+}, \mathbb{R}\right)$, function $f: \mathbb{R}^{m} \times \mathbb{R} \rightarrow$ $\mathbb{R}^{m \times r}$ is continuous, where $r$ and $m$ are positive integers. Finally, $g: \mathbb{R} \rightarrow \mathbb{R}^{r}$ is continuous and satisfies $g(0)=\mathbf{0}$. Observe that if $u$ is constant; then $x(t)=x_{0}$, for all $t \geq 0$. For this reason, we consider only nonconstant inputs $u$ in this paper.

Since $g$ is continuous and $\dot{u} \in L^{\infty}\left(\mathbb{R}_{+}, \mathbb{R}\right)$, we have $g \circ \dot{u} \in L^{\infty}\left(\mathbb{R}, \mathbb{R}^{r}\right)$. The differential equation (1) satisfies Carathéodory conditions, thus, for each initial state $x_{0} \in \mathbb{R}^{m}$, (1) has an absolutely continuous solution that is defined on an interval of the form $[0, T), T>0[21$, page 4$]$.

Consider the time scale change $s_{\gamma}(t)=t / \gamma, \gamma>0, t \geq 0$. When the input $u \circ s_{\gamma}$ is used instead of $u$, the system (1)-(2) becomes

$$
\begin{gathered}
\dot{x}_{\gamma}(t)=f\left(x_{\gamma}(t), u \circ s_{\gamma}(t)\right) g\left(\frac{1}{\gamma} \dot{u} \circ s_{\gamma}(t)\right), \\
x_{\gamma}(0)=x_{0},
\end{gathered}
$$


where $x_{\gamma}$ is the maximal solution of (3). When $\gamma=1$, system (3)-(4) reduces to (1)-(2). For any $\gamma>0$, define $\sigma_{\gamma}: \mathbb{R}_{+} \rightarrow$ $\mathbb{R}^{m}$ as $\sigma_{\gamma}=x_{\gamma} \circ s_{1 / \gamma}$. System (3)-(4) can be rewritten as

$$
\sigma_{\gamma}(t)=x_{0}+\gamma \int_{0}^{t} f\left(\sigma_{\gamma}(\tau), u(\tau)\right) g\left(\frac{1}{\gamma} \dot{u}(\tau)\right) d \tau
$$

for all $\gamma>0$ and for almost all $t \in\left[0, \omega_{\gamma}\right)$, where $\left[0, \omega_{\gamma}\right)$ is the maximal interval of existence of the solution $\sigma_{\gamma}$.

Observe that Lemma 3 implies that for any $\gamma>0$ there exists a unique function $x_{u \circ s_{\gamma}} \in L^{\infty}\left(I_{u}, \mathbb{R}^{m}\right)$ such that $x_{u \circ s_{\gamma}} \circ \rho_{u \circ s_{\gamma}}=x_{\gamma}$ (when $\gamma=1$, we get $x_{u} \circ \rho_{u}=x$ ). The latter equality is equivalent to $x_{u \circ s_{\gamma}} \circ \rho_{u}=\sigma_{\gamma}$. According to Definition 4, the system (1)-(2) is consistent with respect to $\left(u, x_{0}\right)$ if and only if the sequence of functions $x_{u \circ s_{\gamma}}$ converges in $L^{\infty}\left(I_{u}, \mathbb{R}^{m}\right)$.

Proposition 5. The system (1)-(2) is consistent with respect to $\left(u, x_{0}\right)$ in the sense of Definition 4 if and only if the sequence of function $\sigma_{\gamma}$ converges in $L^{\infty}\left(\mathbb{R}_{+}, \mathbb{R}^{m}\right)$ as $\gamma \rightarrow \infty$.

Proof. To prove the if part, define the causal operator $\mathscr{H}$ : $W^{1, \infty}\left(\mathbb{R}_{+}, \mathbb{R}^{n}\right) \times \mathbb{R}^{m} \rightarrow L^{\infty}\left(\mathbb{R}_{+}, \mathbb{R}^{m}\right)$ that maps $\left(u, x_{0}\right)$ to $x$, where $x$ is given in (1)-(2). Assume that there exists $\sigma^{*} \in L^{\infty}\left(\mathbb{R}_{+}, \mathbb{R}^{m}\right)$ such that $\lim _{\gamma \rightarrow \infty}\left\|\sigma_{\gamma}-\sigma^{*}\right\|_{\infty}=0$. We know from (5), that $\sigma_{\gamma}$ is a sequence of continuous functions. Thus, the function $\sigma^{*}$ is continuous as a uniform limit of continuous function. Lemma 3 implies that there exists a unique continuous function $x_{u}^{*} \in L^{\infty}\left(I_{u}, \mathbb{R}^{m}\right)$ such that $x_{u}^{*} \circ \rho_{u}=\sigma^{*}$. Let $\varrho \in I_{u}$. Since $\rho_{u}$ is continuous, there exists some $t \geq 0$ such that $\varrho=\rho_{u}(t)$. We get from the relation $\sigma_{\gamma}=x_{u \circ s_{\gamma}} \circ \rho_{u}$ that for all $\gamma>0: \mid x_{u \circ s_{\gamma}}(\varrho)-$ $x_{u}^{*}(\varrho)|=| x_{u \circ s_{\gamma}} \circ \rho_{u}(t)-x_{u}^{*} \circ \rho_{u}(t) \mid \leq\left\|\sigma_{\gamma}-\sigma^{*}\right\|_{\infty}$. This implies that $\left\|x_{u \circ s_{\gamma}}-x_{u}^{*}\right\|_{\infty, I_{u}} \leq\left\|\sigma_{\gamma}-\sigma^{*}\right\|_{\infty}$ so that $\lim _{\gamma \rightarrow \infty}\left\|x_{u \circ s_{\gamma}}-x_{u}^{*}\right\|_{\infty, I_{u}}=0$, which means that the system (1)-(2) is consistent with respect to $\left(u, x_{0}\right)$.

To prove the only if part, assume that $\lim _{\gamma \rightarrow \infty}\left\|x_{u \circ s_{\gamma}}-x_{u}^{*}\right\|_{\infty, I_{u}}=0$, then the relation $x_{u \circ s_{\gamma}} \circ \rho_{u}=\sigma_{\gamma}$ implies that for almost all $t \geq 0$

$$
\begin{aligned}
\left|\sigma_{\gamma}(t)-x_{u}^{*} \circ \rho_{u}(t)\right| & =\left|x_{u \circ s_{\gamma}} \circ \rho_{u}(t)-x_{u}^{*} \circ \rho_{u}(t)\right| \\
& \leq\left\|x_{u \circ s_{\gamma}}-x_{u}^{*}\right\|_{\infty, I_{u}} .
\end{aligned}
$$

Thus, we have $\left\|\sigma_{\gamma}-x_{u}^{*} \circ \rho_{u}\right\|_{\infty} \leq\left\|x_{u \circ s_{\gamma}}-x_{u}^{*}\right\|_{\infty, I_{u}}$ so that $\lim _{\gamma \rightarrow \infty}\left\|\sigma_{\gamma}-x_{u}^{*} \circ \rho_{u}\right\|_{\infty}=0$.

Proposition 5 implies that the consistency of the system (1)-(2) can be investigated by studying the uniform convergence of the sequence of functions $\sigma_{\gamma}$ instead of $x_{u \circ s_{\gamma}}$. Thus, we know from Section 2 that the system (1)-(2) is a hysteresis only if $\sigma_{\gamma}$ converges uniformly as $\gamma \rightarrow \infty$.

Problem. In this paper, our objective is to derive necessary conditions and sufficient ones for the uniform convergence of the sequence of functions $\sigma_{\gamma}$ as $\gamma \rightarrow \infty$.

\section{Classification of Function $g$}

This section introduces a classification for the function $g$ that is used throughout the paper.

Definition 6. Let $G \in C^{0}\left(\left[t_{1}, t_{2}\right], \mathbb{R}\right)\left(C^{0}\left(\left[t_{1}, t_{2}\right], \mathbb{R}\right)=\{p\right.$ : $\left[t_{1}, t_{2}\right] \rightarrow \mathbb{R}$ such that $p$ is continuous on $\left.\left.\left[t_{1}, t_{2}\right]\right\}\right)$. The right and left local fractional derivatives of $G$ at $t_{3} \in\left(t_{1}, t_{2}\right)$ with respect to order $\lambda>0$ are defined respectively as follows [22]:

$$
\begin{aligned}
& d_{+}^{\lambda} G\left(t_{3}\right)=\Gamma(1+\lambda) \lim _{\kappa \rightarrow t_{3}+} \frac{G(\kappa)-G\left(t_{3}\right)}{\left(\kappa-t_{3}\right)^{\lambda}} \in \mathbb{R}, \\
& d_{-}^{\lambda} G\left(t_{3}\right)=\Gamma(1+\lambda) \lim _{\kappa \rightarrow t_{3}-} \frac{G\left(t_{3}\right)-G(\kappa)}{\left(t_{3}-\kappa\right)^{\lambda}} \in \mathbb{R},
\end{aligned}
$$

where $\Gamma$ is the gamma function.

The local fractional derivative of a vector-valued function is the vector of local fractional derivatives of its components.

Definition 7. The function $g \in C^{0}\left(\mathbb{R}, \mathbb{R}^{r}\right)$ is said to be of class $\lambda>0$ if $g(0)=\mathbf{0}$ and the quantities $d_{+}^{\lambda} g(0)$ and $d_{-}^{\lambda} g(0)$ exist, are finite, and at least one of them is nonzero.

Proposition 8. The function $g \in C^{0}\left(\mathbb{R}, \mathbb{R}^{r}\right)$ is of class $\lambda$ if and only if

$$
\forall \vartheta \in \mathbb{R}, \quad \text { one has } \lim _{\gamma \rightarrow \infty} \gamma^{\lambda} g\left(\frac{\vartheta}{\gamma}\right)=g^{*}(\vartheta) \text {, }
$$

where $g^{*} \in C^{0}\left(\mathbb{R}, \mathbb{R}^{r}\right)$ is defined as

$$
g^{*}(\vartheta)= \begin{cases}\vartheta^{\lambda} \lim _{\kappa \rightarrow 0+} \frac{g(\kappa)}{\kappa^{\lambda}} & \vartheta \geq 0, \\ (-\vartheta)^{\lambda} \lim _{\kappa \rightarrow 0-} \frac{g(\kappa)}{(-\kappa)^{\lambda}} & \vartheta<0 .\end{cases}
$$

Proof. Immediate using of the change of variables $\kappa=\vartheta / \gamma$.

Proposition 9. If the function $g \in C^{0}\left(\mathbb{R}, \mathbb{R}^{r}\right)$ is of class $\lambda$; then $\lim _{\gamma \rightarrow \infty}\left\|\gamma^{\lambda} g(\dot{u} / \gamma)-g^{*}(\dot{u})\right\|_{\infty}=0$, where $g^{*} \in C^{0}\left(\mathbb{R}, \mathbb{R}^{r}\right)$ is defined in (9).

Proof. The result is trivial when $u$ is constant. Assume that $u$ is nonconstant. Given $\varepsilon>0$. Since $g \in C^{0}\left(\mathbb{R}, \mathbb{R}^{r}\right)$ is of class $\lambda$, there exists some $c_{\varepsilon}$; that depend solely on $\varepsilon$, such that

$$
\begin{aligned}
& \left|\frac{g(\vartheta)}{\vartheta^{\lambda}}-\frac{d_{+}^{\lambda} g(0)}{\Gamma(1+\lambda)}\right|<\frac{\varepsilon}{\|\dot{u}\|_{\infty}^{\lambda}}, \quad \text { whenever } 0<\vartheta<c_{\varepsilon}, \\
& \left|\frac{g(\vartheta)}{(-\vartheta)^{\lambda}}-\frac{d_{-}^{\lambda} g(0)}{\Gamma(1+\lambda)}\right|<\frac{\varepsilon}{\|\dot{u}\|_{\infty}^{\lambda}}, \quad \text { whenever }-c_{\varepsilon}<\vartheta<0 .
\end{aligned}
$$


The boundedness of $\dot{u}$ implies that there exists a positive constant $\gamma^{*}$ such that $|\dot{u}(t)| / \gamma<c_{\varepsilon}$, for all $\gamma>\gamma^{*}$. Thus, we have for all $\gamma>\gamma^{*}$,

$$
\begin{aligned}
& \left|\gamma^{\lambda} \frac{g(\dot{u} / \gamma)}{(\dot{u})^{\lambda}}-\frac{d_{+}^{\lambda} g(0)}{\Gamma(1+\lambda)}\right|<\frac{\varepsilon}{\|\dot{u}\|_{\infty}^{\lambda}}, \quad \text { whenever } \dot{u}>0, \\
& \left|\gamma^{\lambda} \frac{g(\dot{u} / \gamma)}{(-\dot{u})^{\lambda}}-\frac{d_{-}^{\lambda} g(0)}{\Gamma(1+\lambda)}\right|<\frac{\varepsilon}{\|\dot{u}\|_{\infty}^{\lambda}}, \quad \text { whenever } \dot{u}<0 .
\end{aligned}
$$

Thus, we get from (9) that

$$
\begin{gathered}
\left|\gamma^{\lambda} g\left(\frac{\dot{u}}{\gamma}\right)-g^{*}(\dot{u})\right| \\
\leq|\dot{u}|^{\lambda} \max \left\{\left|\gamma^{\lambda} \frac{g(\dot{u})}{|\dot{u}|^{\lambda}}-\frac{d_{+}^{\lambda} g(0)}{\Gamma(1+\lambda)}\right|,\right. \\
\left.\left|\gamma^{\lambda} \frac{g(\dot{u})}{|\dot{u}|^{\lambda}}-\frac{d_{-}^{\lambda} g(0)}{\Gamma(1+\lambda)}\right|\right\}<\varepsilon,
\end{gathered}
$$$$
\forall \gamma>\gamma^{*}
$$

which completes the proof.

Proposition 10. If the function $g \in C^{0}\left(\mathbb{R}, \mathbb{R}^{r}\right)$ is of class $\lambda_{1}$ for some $\lambda_{1}>0$; then it cannot be of any class $\lambda_{2}>0$ different than $\lambda_{1}$.

Proof. Assume that the function $g \in C^{0}\left(\mathbb{R}, \mathbb{R}^{r}\right)$ is of class $\lambda_{1}$ and $\lambda_{2}$ with $\lambda_{1}<\lambda_{2}$. Then,

$$
\begin{aligned}
\frac{d_{+}^{\lambda_{1}} g(0)}{\Gamma\left(1+\lambda_{1}\right)} & =\lim _{\kappa \rightarrow 0+} \frac{g(\kappa)}{\kappa^{\lambda_{1}}}=\lim _{\kappa \rightarrow 0+} \kappa^{\lambda_{2}-\lambda_{1}} \frac{g(\kappa)}{\kappa^{\lambda_{2}}} \\
& =0 \times \frac{d_{+}^{\lambda_{2}} g(0)}{\Gamma\left(1+\lambda_{2}\right)}=\mathbf{0}, \\
\frac{d_{-}^{\lambda_{1}} g(0)}{\Gamma\left(1+\lambda_{1}\right)} & =\lim _{\kappa \rightarrow 0-} \frac{g(\kappa)}{(-\kappa)^{\lambda_{1}}}=\lim _{\kappa \rightarrow 0-}|\kappa|^{\lambda_{2}-\lambda_{1}} \frac{g(\kappa)}{|\kappa|^{\lambda_{2}}} \\
& =0 \times \frac{d_{-}^{\lambda_{2}} g(0)}{\Gamma\left(1+\lambda_{2}\right)}=\mathbf{0},
\end{aligned}
$$

which contradicts the fact that $g$ is of class $\lambda_{1}$.

Proposition 11. If the function $g \in C^{0}\left(\mathbb{R}, \mathbb{R}^{r}\right)$ is of class $\lambda>0$; then there exists $g_{0} \in C^{0}\left(\mathbb{R}, \mathbb{R}_{+}\right)$, such that

$$
\left|\gamma^{\lambda} g\left(\frac{\vartheta}{\gamma}\right)\right| \leq g_{0}(\vartheta), \quad \forall \gamma>1, \forall \vartheta \in \mathbb{R} .
$$

Proof. see Appendix A.

\section{Necessary Conditions}

The objective of this section is to derive necessary conditions for the uniform convergence of the sequence of functions $\sigma_{\gamma}$ as $\gamma \rightarrow \infty$.
The standard way to ensure that the system (1)-(2) admits a unique solution is to prove that the right-hand side of (1)-(2) is Lipschitz with respect to $x$. A function $v: D \subseteq \mathbb{R}^{m} \times \mathbb{R}_{+} \rightarrow$ $\mathbb{R}^{m}$ is Lipschitz with respect to $x$ if there exists a summable function $l: \mathbb{R}_{+} \rightarrow \mathbb{R}_{+}$such that $\left|\nu\left(\alpha_{1}, t\right)-\nu\left(\alpha_{2}, t\right)\right| \leq l(t) \mid \alpha_{1}-$ $\alpha_{2} \mid$, for almost all $t \geq 0$ and for all $\alpha_{1}, \alpha_{2} \in \mathbb{R}^{m}$ that satisfy $\left(t, \alpha_{1}\right),\left(t, \alpha_{2}\right) \in D[21]$.

Lemma 12. Assume that the system (1)-(2) has a unique global solution for each input $u \in W^{1, \infty}\left(\mathbb{R}_{+}, \mathbb{R}\right)$ and initial condition $x_{0} \in \mathbb{R}^{m}$. Assume that the function $g$ is of class $\lambda>0$. Suppose that there exists a continuous function $Q: \mathbb{R}_{+} \times \mathbb{R}_{+} \times \mathbb{R}_{+} \rightarrow \mathbb{R}_{+}$ such that

$$
|x(t)| \leq Q\left(\left|x_{0}\right|,\|u\|_{\infty},\|\dot{u}\|_{\infty}\right), \quad \forall t \geq 0,
$$

for each initial state $x_{0} \in \mathbb{R}^{m}$ and each input $u \in W^{1, \infty}\left(\mathbb{R}_{+}, \mathbb{R}\right)$. Assume that the system (1)-(2) is consistent with respect to $\left(u, x_{0}\right)$; that is, there exists $q_{u} \in L^{\infty}\left(\mathbb{R}_{+}, \mathbb{R}^{m}\right)$ such that $\lim _{\gamma \rightarrow \infty}\left\|\sigma_{\gamma}-q_{u}\right\|_{\infty}=0$ (see Proposition 5); then

$$
\text { If } \lambda=1 \text {, one has }
$$

(i) $q_{u} \in W^{1, \infty}\left(\mathbb{R}_{+}, \mathbb{R}^{m}\right)$.

(ii) one has for all $t \geq 0$ that

$$
q_{u}(t)=x_{0}+\int_{0}^{t} f\left(q_{u}(\tau), u(\tau)\right) g^{*}(\dot{u}(\tau)) d \tau,
$$

where $g^{*}$ is given in (9).

If $\lambda \in(0,1)$, one has

(i) $q_{u} \in C^{0}\left(\mathbb{R}_{+}, \mathbb{R}^{m}\right) \cap L^{\infty}\left(\mathbb{R}_{+}, \mathbb{R}^{m}\right)$.

(ii) $q_{u}(0)=x_{0}$.

(iii) one has for almost all $t \geq 0$ that

$$
f\left(q_{u}(t), u(t)\right) g^{*}(\dot{u}(t))=\mathbf{0},
$$

where $g^{*}$ is defined in (9).

$$
\text { If } \lambda>1 \text {, one has } q_{u}(t)=x_{0} \text {, for all } t \geq 0 \text {. }
$$

Proof. By (15), the fact that $u \in W^{1, \infty}\left(\mathbb{R}_{+}, \mathbb{R}^{n}\right)$, the continuity of the function $Q$, and the relation $\|u\|_{\infty}=\left\|u \circ s_{\gamma}\right\|_{\infty}$, for all $\gamma>0$, there exists some $a>0$ independent of $\gamma$, and $\gamma_{0}>0$ such that

$$
\left|x_{\gamma}(t)\right| \leq Q\left(\left|x_{0}\right|,\|u\|_{\infty}, \frac{\|\dot{u}\|_{\infty}}{\gamma}\right) \leq a, \quad \forall t \geq 0, \forall \gamma>\gamma_{0},
$$

where $x_{\gamma}$ is given in (3)-(4). Thus,

$$
\left\|x_{\gamma}\right\|_{\infty} \leq a, \quad \forall \gamma>\gamma_{0} .
$$

On the other hand, we conclude from Lemma 3 that $x_{u \circ s_{\gamma}} \in$ $C^{0}\left(I_{u}, \mathbb{R}^{m}\right) \cap L^{\infty}\left(I_{u}, \mathbb{R}^{m}\right)$ and $\left\|x_{u \circ s_{\gamma}}\right\|_{\infty, I_{u}}=\left\|x_{\gamma}\right\|_{\infty}$, for all $\gamma>$ 0 . Hence, the continuity of $x_{u \circ s_{\gamma}}$ and (19) imply that

$$
\begin{array}{r}
\left|\sigma_{\gamma}(t)\right|=\left|x_{u \circ s_{\gamma}} \circ \rho_{u}(t)\right| \leq\left\|x_{u \circ s_{\gamma}}\right\|_{\infty, I_{u}}=\left\|x_{\gamma}\right\|_{\infty} \leq a, \\
\forall t \geq 0, \quad \forall \gamma>\gamma_{0} .
\end{array}
$$


Thus, the continuity of $f$ and $g$, the boundedness of $\dot{u}$, and Proposition 11, imply that there exists a constant $b>0$ independent of $\gamma$ such that $\gamma^{\lambda}\left|f\left(\sigma_{\gamma}(\tau), u(\tau)\right) g(\dot{u}(\tau) / \gamma)\right| \leq b$, for almost all $\tau \geq 0$, for all $\gamma>1$. Thus, we can apply the Dominated Lebesgue Theorem [23] to get

$$
\begin{aligned}
\lim _{\gamma \rightarrow \infty} \gamma^{\lambda} \int_{0}^{t} f\left(\sigma_{\gamma}(\tau), u(\tau)\right) g\left(\frac{\dot{u}(\tau)}{\gamma}\right) d \tau \\
\quad=\int_{0}^{t} f\left(x_{u}^{*} \circ \rho_{u}(\tau), u(\tau)\right) g^{*}(\dot{u}(\tau)) d \tau, \quad \forall t \geq 0 .
\end{aligned}
$$

On the other hand, since $q_{u}$ is continuous as a uniform limit of continuous sequence of functions, we have $q_{u} \in$ $C^{0}\left(\mathbb{R}_{+}, \mathbb{R}^{m}\right) \cap L^{\infty}\left(\mathbb{R}_{+}, \mathbb{R}^{m}\right)$ and $q_{u}(0)=x_{0}$ (note that $\sigma_{\gamma}(0)=$ $x_{0}$, for all $\left.\gamma>0\right)$.

When $\lambda=1$, we obtain from (21) and (5) that $q_{u}(t)=x_{0}+$ $\int_{0}^{t} f\left(q_{u}(\tau), u(\tau)\right) g^{*}(\dot{u}(\tau)) d \tau$, for all $t \geq 0$. Thus, the continuity of the functions $f$ and $g^{*}$ along with the boundedness of the functions $q_{u}, u$, and $\dot{u}$ implies that the function $\dot{q}_{u}$ is bounded. Therefore, $q_{u} \in W^{1, \infty}\left(\mathbb{R}_{+}, \mathbb{R}^{m}\right)$ and (16) is satisfied.

When $\lambda \in(0,1)$, we get from inequality (20) that $\left\|\sigma_{\gamma}-x_{0}\right\|_{\infty} / \gamma^{1-\lambda} \rightarrow 0$ as $\gamma \rightarrow \infty$. Moreover, (5) can be written for all $t \geq 0$ as

$$
\frac{\sigma_{\gamma}(t)-x_{0}}{\gamma^{1-\lambda}}=\gamma^{\lambda} \int_{0}^{t} f\left(\sigma_{\gamma}(\tau), u(\tau)\right) g\left(\frac{\dot{u}(\tau)}{\gamma}\right) d \tau .
$$

The fact that $\lim _{\gamma \rightarrow 0}\left\|\sigma_{\gamma}-x_{0}\right\|_{\infty} / \gamma^{1-\lambda}=0$, along with (21) and (22) implies that

$$
\int_{0}^{t} f\left(x_{u}^{*} \circ \rho_{u}(\tau), u(\tau)\right) g^{*}(\dot{u}(\tau)) d \tau=\mathbf{0}, \quad \forall t \geq 0,
$$

which proves (17).

Finally, when $\lambda>1$, (5) implies for all $t \geq 0$ that

$$
\sigma_{\gamma}(t)-x_{0}=\gamma^{1-\lambda} \gamma^{\lambda} \int_{0}^{t} f\left(\sigma_{\gamma}(\tau), u(\tau)\right) g\left(\frac{\dot{u}(\tau)}{\gamma}\right) d \tau,
$$

and thus, we get from (21) that $\lim _{\gamma \rightarrow \infty} \sigma_{\gamma}(t)=x_{0}$, for all $t \geq 0$. Therefore, the uniqueness of limits and the continuity of $q_{u}$ imply that $q_{u}(t)=x_{0}$, for all $t \geq 0$.

Remark 13. Observe that for $\lambda>1$, the fact that $q_{u}(t)=$ $x_{0}$, for all $t \geq 0$, means that system(1)-(2)does not represent a hysteresis behavior [24].

Remark 14. For the case $\lambda \in(0,1),(17)$ and the fact that $q_{u}(0)=x_{0}$ imply that $f\left(x_{0}, u(0)\right) g(\dot{u}(0))=\mathbf{0}$, whenever $\dot{u}(0)$ exists.

Example 15. Consider the Following LuGre model [10]:

$$
\begin{aligned}
& \dot{x}=\dot{u}-\theta \frac{|\dot{u}|}{\zeta(\dot{u})} x=\left[\begin{array}{ll}
1 & x
\end{array}\right]\left[\begin{array}{c}
\dot{u} \\
-\theta \frac{|\dot{u}|}{\zeta(\dot{u})}
\end{array}\right]=f(x) g(\dot{u}), \\
& x(0)=x_{0} \text {, }
\end{aligned}
$$

where parameter $\theta$ is the stiffness, $x \in \mathbb{R}$ is the average deflection of the bristles and is the output of the system, $x_{0} \in \mathbb{R}$ is the initial condition, $u \in W^{1, \infty}\left(\mathbb{R}_{+}, \mathbb{R}\right)$ is the relative displacement, and is the input of the system. The function $\zeta: \mathbb{R} \rightarrow \mathbb{R}$ is defined as

$$
\zeta(\alpha)=F_{C}+\left(F_{S}-F_{C}\right) e^{-\left|\alpha / v_{s}\right|}, \quad \forall \alpha \in \mathbb{R},
$$

where $F_{C}>0$ is the Coulomb friction force, $F_{S}>0$ is the stiction force, and $v_{s}>0$ is the Stribeck velocity.

System (25)-(26) has a unique global solution [21, page 5]. On the other hand, the sequence of function $\sigma_{\gamma}$ is given by (see (5))

$$
\dot{\sigma}_{\gamma}(t)=\dot{u}(t)-\theta \frac{|\dot{u}(t)|}{\zeta(\dot{u}(t) / \gamma)} \sigma_{\gamma}(t), \quad \text { for almost all } t \geq 0
$$

The following facts are proved in Example 29.

(i) There exist $\gamma_{1}, E>0$ such that $\left|x_{\gamma}(t)\right| \leq E$, for all $\gamma>$ $\gamma_{1}$, where $x_{\gamma}$ is the output when we use input $u \circ s_{\gamma}$ instead of $u$ (see system (3)-(4)).

(ii) $\left\|\sigma_{\gamma}-q_{u}\right\|_{\infty} \rightarrow 0$ as $\gamma \rightarrow \infty$, where the function $q_{u} \in W^{1, \infty}\left(\mathbb{R}_{+}, \mathbb{R}^{m}\right)$ is defined for all $t \geq 0$ as

$$
\begin{aligned}
q_{u}(t)= & x_{0} \\
& +\int_{0}^{t}\left(\dot{u}(\tau)-\frac{\theta|\dot{u}(\tau)|}{F_{S}} q_{u}(\tau)\right) d \tau .
\end{aligned}
$$

Thus, all conditions of Lemma 12 are satisfied.

Now, we have to find the value of $\lambda$ and the function $g^{*}$. We have

$$
\begin{gathered}
\lim _{\kappa \rightarrow 0+} \frac{g(\kappa)}{\kappa}=\left[\begin{array}{c}
1 \\
\frac{-\theta}{F_{S}}
\end{array}\right], \\
\lim _{\kappa \rightarrow 0-} \frac{g(\kappa)}{(-\kappa)}=\left[\begin{array}{c}
-1 \\
\frac{-\theta}{F_{S}}
\end{array}\right] .
\end{gathered}
$$

Thus, the function $g \in C^{0}\left(\mathbb{R}_{+}, \mathbb{R}^{r}\right)$ in (25) is of class $\lambda=1$ (see Definition 7) and the function $g^{*} \in C^{0}\left(\mathbb{R}_{+}, \mathbb{R}^{r}\right)$ in (9) is defined as

$$
g^{*}(\vartheta)=\left[\begin{array}{c}
\vartheta \\
\frac{-\theta}{F_{S}}|\vartheta|
\end{array}\right], \quad \forall \vartheta \in \mathbb{R}
$$

Therefore, by applying Lemma 12, it follows that the system (29) satisfies (16).

Simulations. Take $\theta=10^{5} \mathrm{~N} / \mathrm{m}, v_{s}=0.001 \mathrm{~m} / \mathrm{s}, F_{S}=1.5 \mathrm{~N}$, $F_{C}=1.0 \mathrm{~N}, x(0)=0 \mathrm{~N}$, and $u(t)=10^{-4} \sin (t) \mathrm{m}$, for all $t \geq 0$ (values taken from [7]). Figure 1(a) shows that the graphs $\left\{\left(\sigma_{\gamma}(t), u(t)\right), t \geq 0\right\}$ converge to the hysteresis loop $\left\{\left(q_{u}(t), u(t)\right), t \geq 0\right\}$ as $\gamma \rightarrow \infty$. This is the 
main characteristic of a hysteresis system. Also, observe that $\left\{\left(\sigma_{\gamma}(t), u(t)\right), t \geq 0\right\}$ are different for different values of $\gamma$. This is what is called "rate-dependent" property of the model (25)(26). Figure 1(b) presents the graph of $\left(\sigma_{\gamma}-q_{u}\right)(t)$ versus $t$; we observe that $\sigma_{\gamma}-q_{u}$ converges uniformly to the zero function as $\gamma \rightarrow \infty$ which means that $\sigma_{\gamma}$ converges uniformly to $q_{u}$ when $\gamma \rightarrow \infty$. The graph of $q_{u}(t)$ versus $t$ is presented in Figure 1(c).

\section{Sufficient Conditions}

This section presents sufficient conditions for the uniform convergence of the sequence of functions $\sigma_{\gamma}$ as $\gamma \rightarrow \infty$ (and hence for consistency of the system (1)-(2) with respect to $\left.\left(u, x_{0}\right)\right)$. The main results of this section are given in Lemmas 20,23 , and 27.

6.1. Class $\lambda \in(0,1)$ Functions. In this subsection, sufficient conditions for the uniform convergence of $\sigma_{\gamma}$ as $\gamma \rightarrow \infty$, are derived when the function $g$ is of class $\lambda \in(0,1)$.

Definition 16 (see [25]). A continuous function $\beta: \mathbb{R}_{+} \rightarrow$ $\mathbb{R}_{+}$is said to belong to class $\mathscr{K}_{\infty}$ if it is increasing, satisfies $\beta(0)=0$, and $\lim _{t \rightarrow \infty} \beta(t)=\infty$.

The following lemma generalizes Theorem 4.18 in [25, page 172]. Indeed, in [25], continuous differentiability is needed, while in Lemma 17, we only need absolute continuity. Also, in [25], the inequality on the derivative of the Lyapunov function is needed everywhere, while in Lemma 17 it is needed only almost everywhere.

Lemma 17. Consider a function $z:[0, \omega) \subseteq \mathbb{R}_{+} \rightarrow \mathbb{R}_{+}$, where $\omega>0$ is finite or infinite. Assume the following.

(1) The function $z$ is absolutely continuous on each compact interval of $[0, \omega)$.

(2) There exist $z_{1} \geq 0$ and $z_{2}>0$ such that $z_{1}<z_{2}, z(0)<$ $z_{2}$ and

$$
\begin{aligned}
\dot{z}(t) \leq 0 & \text { for almost all } t \in[0, \omega) \\
& \text { that satisfy } z_{1}<z(t)<z_{2} .
\end{aligned}
$$

Then, $z(t) \leq \max \left(z(0), z_{1}\right)$, for all $t \in[0, \omega)$.

Proof. see Appendix B.

Example 18. We want to study the stability of the following system

$$
\begin{gathered}
\dot{x}(t)=-x^{3}(t)+u(t), \\
x(0)=x_{0},
\end{gathered}
$$

where $x_{0}$ and state $x$ take values in $\mathbb{R}$, and input $u \in$ $W^{1, \infty}\left(\mathbb{R}_{+}, \mathbb{R}\right)$. System (33) has an absolutely continuous solution that is defined on an interval of the form $[0, \omega)[21$, page 4].

Let $z:[0, \omega) \rightarrow \mathbb{R}_{+}$be such that $z(t)=x^{2}(t)$, for all $t \epsilon$ $[0, \omega)$. The function $z$ is absolutely continuous on each compact subset of $[0, \omega)$ because $x$ is absolutely continuous. Thus, condition (1) in Lemma 17 is satisfied.

We have for almost all $t \in[0, \omega)$ that

$$
\begin{aligned}
\dot{z}(t) & =2 x(t) \cdot \dot{x}(t)=2 x(t)\left(-x^{3}(t)+u(t)\right) \\
& \leq-2 z^{2}(t)+2\|u\|_{\infty} \sqrt{z(t)}
\end{aligned}
$$

Thus,

$$
\begin{array}{ll}
\dot{z}(t) \leq 0 & \text { for almost all } t \in[0, \omega) \\
& \text { that satisfy }\|u\|_{\infty}^{2 / 3}<z(t) .
\end{array}
$$

Therefore, condition (2) in Lemma 17 is satisfied with $z_{1}=\|u\|_{\infty}^{2 / 3}$ and $z_{2}$ can be any positive real number such that $z_{2}>\max \left(z(0), z_{1}\right)=\max \left(x_{0}^{2},\|u\|_{\infty}^{2 / 3}\right)$. Thus, we deduce from Lemma 17 that $z(t) \leq \max \left(z(0),\|u\|_{\infty}^{2 / 3}\right)=$ $\max \left(x_{0}^{2},\|u\|_{\infty}^{2 / 3}\right)$, for all $t \in[0, \omega)$, and hence $|x(t)| \leq$ $\max \left(\left|x_{0}\right|, \sqrt[3]{\|u\|_{\infty}}\right)$, for all $t \in[0, \omega)$.

Corollary 19. Consider a function $z:[0, \omega) \subseteq \mathbb{R}_{+} \rightarrow \mathbb{R}_{+}$, where $\omega>0$ may be infinite. Assume the following.

(1) The function $z$ is absolutely continuous on each compact subset of $[0, \omega)$.

(2) There exist a class $\mathscr{K}_{\infty}$ function $\beta: \mathbb{R}_{+} \rightarrow \mathbb{R}_{+}$and constants $z_{1} \geq 0, z_{2}>0$, and $z_{3} \geq 0$ such that $\max \left\{\beta^{-1}\left(z_{3}\right), z_{1}, z(0)\right\}<z_{2}$, and

$$
\begin{aligned}
\dot{z}(t) \leq & -\beta(z(t)) \\
& +z_{3} \quad \text { for almost all } t \in[0, \omega) \\
& \text { that satisfy } z_{1}<z(t)<z_{2} .
\end{aligned}
$$

Then, $z(t) \leq \max \left\{z(0), z_{1}, \beta^{-1}\left(z_{3}\right)\right\}$, for all $t \in[0, \omega)$.

Proof. We have from (36) that

$$
\begin{aligned}
& \dot{z}(t) \leq 0 \quad \text { for almost all } t \in[0, \omega) \\
& \text { that satisfy } \max \left\{\beta^{-1}\left(z_{3}\right), z_{1}\right\}<z(t)<z_{2},
\end{aligned}
$$

and hence the result follows directly from Lemma 17.

Although the latter corollary follows immediately from Lemma 17, it is useful in many situations [25]. 


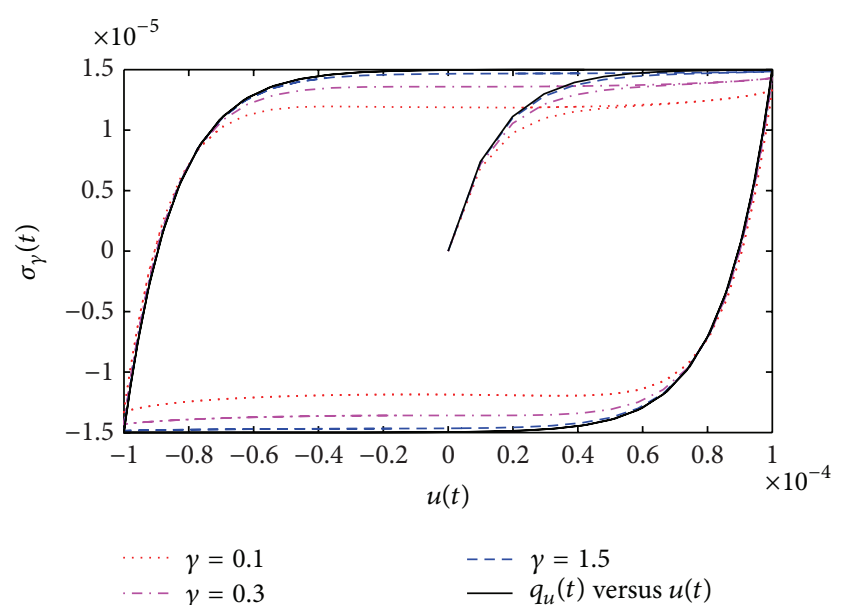

(a)

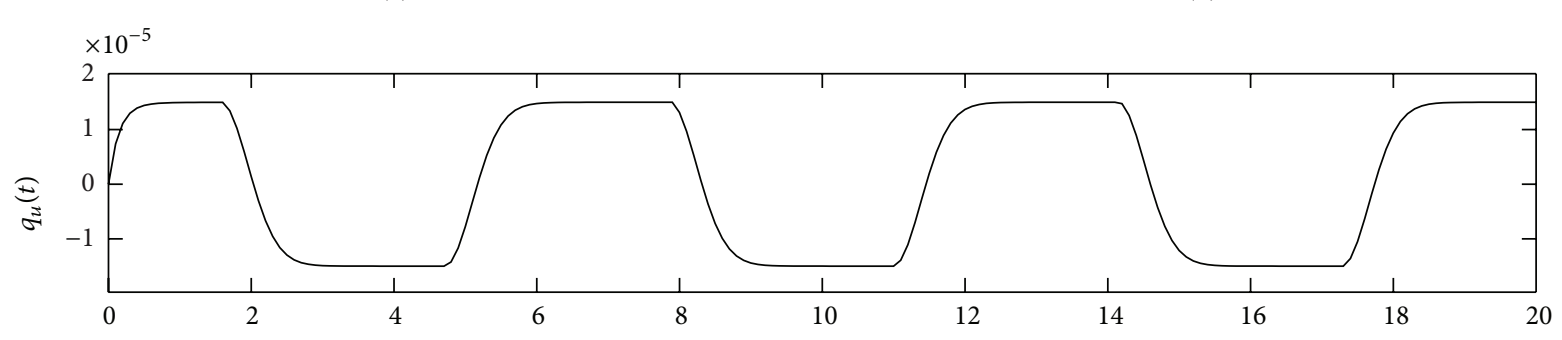

(c)
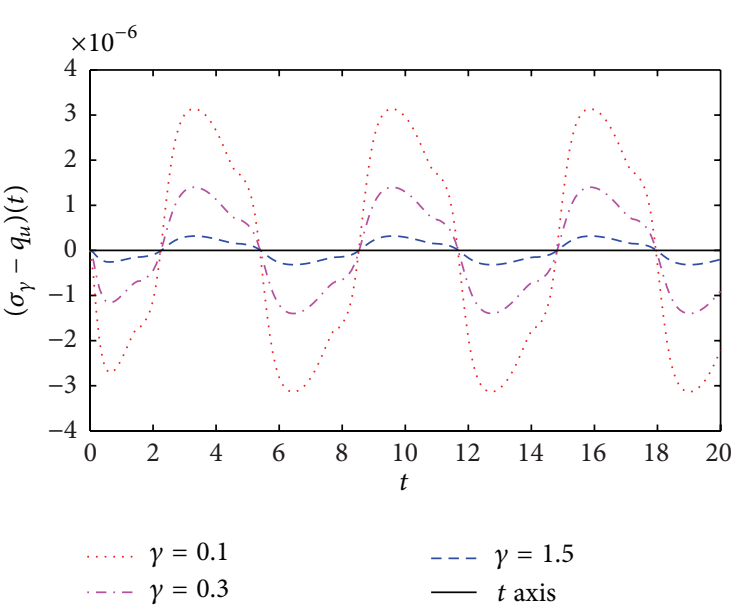

(b)

FiguRE 1: (a) $\sigma_{\gamma}(t)$ versus $u(t)$ for different values of $\gamma$. (b) $\left(\sigma_{\gamma}-q_{u}\right)(t)$ versus $t$ for different values of $\gamma$. (c) $q_{u}(t)$ versus $t$. The plots are for the LuGre model (25)-(26), where the solid lines are for $q_{u}$.

Lemma 20. Suppose that the system (1)-(2) has a unique solution and that the function $g$ is of class $\lambda \in(0,1)$. Assume that there exists $q_{u} \in W^{1, \infty}\left(\mathbb{R}_{+}, \mathbb{R}^{m}\right)$ such that for almost all $t \geq 0$

$$
\begin{gathered}
q_{u}(0)=x_{0}, \\
f\left(q_{u}(t), u(t)\right) g^{*}(\dot{u}(t))=\mathbf{0} .
\end{gathered}
$$

For all $\gamma>0$, define $y_{\gamma}: \mathbb{R}_{+} \rightarrow \mathbb{R}^{m}$ as

$$
y_{\gamma}(t)=\sigma_{\gamma}(t)-q_{u}(t)=x_{\gamma}(\gamma t)-q_{u}(t)
$$

for all $t \in\left[0, \omega_{\gamma}\right)$, where $\left[0, \omega_{\gamma}\right)$ is the maximal interval of existence of solution $\sigma_{\gamma}$ in (5). Suppose that we can find a continuously differentiable function $V: \mathbb{R}^{m} \rightarrow \mathbb{R}_{+}$such that

(1) there exists a function $\delta_{1}: \mathbb{R}_{+} \rightarrow \mathbb{R}_{+}$that satisfies

$$
\delta_{1}(\gamma) \longrightarrow 0 \text { as } \gamma \longrightarrow \infty
$$

(2) there exist constants $\gamma_{*}, \delta_{2}>0$, continuous functions $R_{1}, R_{2}: \mathbb{R}_{+} \rightarrow \mathbb{R}_{+}$and $\mathscr{K}_{\infty}$ class functions $\beta_{1}, \beta_{2}, \beta_{3}:$ $\mathbb{R}_{+} \rightarrow \mathbb{R}_{+}$, satisfying

$$
\beta_{1}(|\alpha|) \leq V(\alpha) \leq \beta_{2}(|\alpha|), \quad \forall \alpha \in \mathbb{R}^{m},
$$

$$
\begin{aligned}
& \left.\frac{d V(\alpha)}{d \alpha}\right|_{\alpha=y_{\gamma}(t)} \cdot f\left(y_{\gamma}(t)+q_{u}(t), u(t)\right) g\left(\frac{\dot{u}(t)}{\gamma}\right) \\
& \leq-\frac{1}{\gamma^{\lambda}} \beta_{3}\left(\left|y_{\gamma}(t)\right|\right) \\
& \quad+\frac{1}{\gamma} R_{1}\left(\left|y_{\gamma}(t)\right|\right) \quad \text { for almost all } t \in\left[0, \omega_{\gamma}\right), \forall \gamma>\gamma_{*} \\
& \text { that satisfy } \delta_{1}(\gamma)<\left|y_{\gamma}(t)\right|<\delta_{2},
\end{aligned}
$$

$$
\left|\frac{d V(\alpha)}{d \alpha}\right| \leq R_{2}(|\alpha|), \quad \forall \alpha \in \mathbb{R}^{m}
$$

Then,

(i) there exist $E, \gamma^{*}>0$ such that for all $\gamma>\gamma^{*}: \omega_{\gamma}=+\infty$ and $\left\|x_{\gamma}\right\|_{\infty} \leq E$, where $x_{\gamma}$ is given in (3)-(4).

(ii) $\lim _{\gamma \rightarrow \infty}\left\|\sigma_{\gamma}-q_{u}\right\|_{\infty}=0$. 
Proof. From (5) and (39), we get for all $\gamma>0$ and almost all $t \in\left[0, \omega_{\gamma}\right)$ that $y_{\gamma}(0)=\mathbf{0}$ and

$$
\dot{y}_{\gamma}(t)=\gamma f\left(y_{\gamma}(t)+q_{u}(t), u(t)\right) g\left(\frac{\dot{u}(t)}{\gamma}\right)-\dot{q}_{u}(t) .
$$

For any $\gamma>0$, define $V_{\gamma}:\left(0, \omega_{\gamma}\right] \rightarrow \mathbb{R}_{+}$as $V_{\gamma}(t)=$ $V\left(y_{\gamma}(t)\right)$, for all $t \in\left(0, \omega_{\gamma}\right]$. Note that function $V_{\gamma}$ is absolutely continuous on each compact subset of $\left[0, \omega_{\gamma}\right)$ as a composition of a continuously differentiable function $V$ and an absolutely continuous function $y_{\gamma}$. The derivative of $V$ along with trajectories (44) is given for almost all $t \in\left[0, \omega_{\gamma}\right)$ and all $\gamma>0$ by

$$
\begin{aligned}
\dot{V}_{\gamma}(t) & =\left.\frac{d V(\alpha)}{d \alpha}\right|_{\alpha=y_{\gamma}(t)} \cdot \dot{y}_{\gamma}(t) \\
& =\left.\frac{d V(\alpha)}{d \alpha}\right|_{\alpha=y_{\gamma}(t)} \cdot\left[\gamma f\left(y_{\gamma}(t)+q_{u}(t), u(t)\right)-\dot{q}_{u}(t)\right] .
\end{aligned}
$$

By (40), there exists some $\gamma_{1}>\gamma_{*}$, such that $\beta_{2} \circ \delta_{1}(\gamma)<$ $\beta_{1}\left(\delta_{2}\right)$, for all $\gamma>\gamma_{1}$. Let $\Omega_{\gamma}=\left(\beta_{2} \circ \delta_{1}(\gamma), \beta_{1}\left(\delta_{2}\right)\right)$. By (41), we have for any $\gamma>\gamma_{1}$, for almost all $t \in\left[0, \omega_{\gamma}\right)$ that

$$
V_{\gamma}(t) \in \Omega_{\gamma} \Longrightarrow \delta_{1}(\gamma)<\left|y_{\gamma}(t)\right|<\delta_{2} .
$$

Thus, we deduce from (42), (43), (45), and (46) that

$$
\begin{aligned}
& \dot{V}_{\gamma}(t) \\
& \leq-\gamma^{1-\lambda} \beta_{3}\left(\left|y_{\gamma}(t)\right|\right)+R_{1}\left(\left|y_{\gamma}(t)\right|\right) \\
& +\left\|\dot{q}_{u}\right\|_{\infty} R_{2}\left(\left|y_{\gamma}(t)\right|\right), \quad \text { for almost all } t \in\left[0, \omega_{\gamma}\right), \forall \gamma>\gamma_{1} \\
& \text { that satisfy } V_{\gamma}(t) \in \Omega_{\gamma} .
\end{aligned}
$$

Therefore, (46) and the continuity of the functions $R_{1}$ and $R_{2}$ imply that there exists a constant $a>0$ that does not depend on $\gamma$, such that

$$
\begin{aligned}
& \dot{V}_{\gamma}(t) \\
& \leq-\gamma^{1-\lambda} \beta_{3}\left(\left|y_{\gamma}(t)\right|\right)+a, \quad \text { for almost all } t \in\left[0, \omega_{\gamma}\right), \forall \gamma>\gamma_{1} \\
& \text { that satisfy } V_{\gamma}(t) \in \Omega_{\gamma} .
\end{aligned}
$$

Thus, we deduce from (41) that

$$
\begin{aligned}
& \dot{V}_{\gamma}(t) \\
& \leq-\beta_{\gamma}\left(V_{\gamma}(t)\right)+a, \quad \text { for almost all } t \in\left[0, \omega_{\gamma}\right), \forall \gamma>\gamma_{1} \\
& \text { that satisfy } V_{\gamma}(t) \in \Omega_{\gamma},
\end{aligned}
$$

where $\beta_{\gamma}: \mathbb{R}_{+} \rightarrow \mathbb{R}_{+}$is defined as $\beta_{\gamma}=\gamma^{1-\lambda} \beta_{3} \circ \beta_{2}^{-1}$.
On the other hand, since $\lambda \in(0,1)$, there exists $\gamma_{2}>\gamma_{1}$ such that $\beta_{\gamma}^{-1}(a)=\beta_{2} \circ \beta_{3}^{-1}\left(a \gamma^{\lambda-1}\right)<$ $\beta_{1}\left(\delta_{2}\right)$, for all $\gamma>\gamma_{2}$. Hence, Corollary 19 and the fact that $V_{\gamma}(0)=0$, for all $\gamma>0$, imply that $V_{\gamma}(t) \leq \max \left\{\beta_{\gamma}^{-1}(a)\right.$, $\left.\beta_{2} \circ \delta_{1}(\gamma)\right\}=\max \left\{\beta_{2} \circ \beta_{3}^{-1}\left(a \gamma^{\lambda-1}\right), \beta_{2} \circ \delta_{1}(\gamma)\right\}$, for all $t \epsilon$ $\left[0, \omega_{\gamma}\right)$ and for all $\gamma>\gamma_{2}$. Therefore, (41) implies for all $\gamma>\gamma_{2}$ and for all $t \in\left[0, \omega_{\gamma}\right)$ that

$$
\begin{gathered}
\left|y_{\gamma}(t)\right| \leq \max \left\{\beta_{1}^{-1} \circ \beta_{2} \circ \beta_{3}^{-1}\left(a \gamma^{\lambda-1}\right),\right. \\
\left.\beta_{1}^{-1} \circ \beta_{2} \circ \delta_{3}(\gamma)\right\} .
\end{gathered}
$$

Thus, $\omega_{\gamma}=+\infty$, for all $\gamma>\gamma_{2}$. Furthermore, (40), (50), and the fact that $\lambda \in(0,1)$ imply that $\left\|y_{\gamma}\right\|_{\infty}=\left\|\sigma_{\gamma}-q_{u}\right\|_{\infty} \rightarrow 0$ as $\gamma \rightarrow \infty$. This proves the consistency with respect to $\left(u, x_{0}\right)$ because of Proposition 5.

Moreover, by (50), there exist some $E>0, \gamma^{*}>\gamma_{2}$, such that

$$
\left\|\sigma_{\gamma}\right\|_{\infty} \leq E, \quad \forall \gamma>\gamma^{*}
$$

On the other hand, let $\gamma>\gamma^{*}$. Since $x_{\gamma}$ is continuous, Lemma 3 ensures that $\left\|x_{u \circ s_{\gamma}}\right\|_{\infty, I_{u}}=\left\|x_{\gamma}\right\|_{\infty}$. Let $\varrho \in I_{u}$. Due to the continuity of $\rho_{u}$, there exists some $t \geq 0$ such that $\varrho=\rho_{u}(t)$ and thus (51) and the continuity of $\sigma_{\gamma}$ lead to $\left|x_{u \circ s_{\gamma}}(\varrho)\right|=\left|x_{u \circ s_{\gamma}} \circ \rho_{u}(t)\right|=\left|\sigma_{\gamma}(t)\right| \leq\left\|\sigma_{\gamma}\right\|_{\infty} \leq E$, for all $\gamma>$ $\gamma^{*}$. Therefore, $\left\|x_{u \circ s_{\gamma}}\right\|_{\infty}=\left\|x_{\gamma}\right\|_{\infty} \leq E$, for all $\gamma>\gamma^{*}$, which completes the proof.

Remark 21. For $\lambda \in(0,1)$,if the function $q_{u} \in C^{0}\left(\mathbb{R}_{+}, \mathbb{R}^{m}\right) \cap$ $L^{\infty}\left(\mathbb{R}_{+}, \mathbb{R}^{m}\right)$ in Lemma 20 , such that $q_{u}=R(u)$ for some $R$ : $\mathbb{R} \rightarrow \mathbb{R}^{m}$, then the graphs $\left\{\left(\sigma_{\gamma}(t), u(t)\right), t \geq 0\right\}$ converge to the curve $R$ as $\gamma \rightarrow \infty$. Hence, (1)-(2) is not a hysteresis because the hysteresis loop cannot be a function [24]. This fact is illustrated in Example 22.

Example 22. Consider the following semilinear Duhem model:

$$
\begin{gathered}
\dot{x}=(A x+B u) g(\dot{u}), \\
x(0)=x_{0},
\end{gathered}
$$

where $A$ is a Hurwitz $m \times m$ matrix (i.e., every eigenvalue of $A$ has a negative real part), vector $B$ and state $x$ take values in $\mathbb{R}^{m}$. The right-hand side of (52) is Lipschitz and thus the system has a unique solution [21]. Take an input $u \in W^{1, \infty}\left(\mathbb{R}_{+}, \mathbb{R}\right)$ such that $A^{-1} B u(0)=-x_{0}$ and that $|\dot{u}(t)| \geq M$ for almost all $t \in \mathbb{R}$ and for some $M>0$. Assume that the function $g: \mathbb{R} \rightarrow \mathbb{R}_{+}$is of class $\lambda \in(0,1)$ and that $d_{+}^{\lambda} g(0), d_{-}^{\lambda} g(0)>0$. Thus, there exists $L>0$ such that $g^{*}(\vartheta) \geq L|\vartheta|^{\lambda}$, for all $\vartheta \in \mathbb{R}$, where the function $g^{*}$ is defined in (9). On the other hand, Proposition 9 states that $\lim _{\gamma \rightarrow \infty}\left\|\gamma^{\lambda} g(\dot{u} / \gamma)-g^{*}(\dot{u})\right\|_{\infty}=0$. This means that there exists $\gamma_{1}>0$ such that we get for almost all $t \geq 0$, and all $\gamma>\gamma_{1}$ that

$$
\gamma^{\lambda} g\left(\frac{\dot{u}(t)}{\gamma}\right)>g^{*}(\dot{u}(t))-\frac{L M^{\lambda}}{2} .
$$


Thus, the facts that $|\dot{u}| \geq M$ and $g^{*}(\cdot) \geq L|\cdot|^{\lambda}$ imply that

$$
g\left(\frac{\dot{u}(t)}{\gamma}\right)>\frac{L M^{\lambda}}{2 \gamma^{\lambda}}, \quad \text { for almost all } t \geq 0, \forall \gamma>\gamma_{1} .
$$

The function $q_{u} \in W^{1, \infty}\left(\mathbb{R}_{+}, \mathbb{R}\right)$ which is defined as $q_{u}=$ $-A^{-1} B u$ satisfies (38) because $A^{-1} B u(0)=-x_{0}$.

Since $A$ is Hurwitz, there exists a $m \times m$ positive-definite matrix $P$ such that [25, page 136]

$$
P A+A^{T} P=-I,
$$

where $I$ is the identity matrix. Consider the continuously differentiable quadratic Lyapunov function candidate $V$ : $\mathbb{R}^{m} \rightarrow \mathbb{R}$ such that $V(\alpha)=\alpha^{T} P \alpha$, for all $\alpha \in \mathbb{R}^{m}$. Since $P$ is symmetric, we have for all $\alpha \in \mathbb{R}^{m}$ that

$$
\lambda_{\min }(P)|\alpha|^{2} \leq V(\alpha)=\alpha^{T} P \alpha \leq \lambda_{\max }(P)|\alpha|^{2},
$$

where $\lambda_{\max }(P)$ and $\lambda_{\min }(P)$ are, respectively, the maximum and minimum eigenvalues of the matrix $P$. This shows that (41) is satisfied with $\beta_{1}(\eta)=\lambda_{\min }(P) \eta^{2}$ and $\beta_{2}(\eta)=$ $\lambda_{\max }(P) \eta^{2}$ for all $\eta>0$. Since $P$ is symmetric, we have the following matrices derivation:

$$
\frac{d V(\alpha)}{d \alpha}=\left(P+P^{T}\right) \alpha=2 P \alpha, \quad \forall \alpha \in \mathbb{R}^{m} .
$$

Thus, we get

$$
\left|\frac{d V(\alpha)}{d \alpha}\right|=2|P \alpha| \leq 2|P||\alpha|, \quad \forall \alpha \in \mathbb{R}^{m},
$$

where $|P|$ is the induced 2-norm for the matrix $P$ and hence (43) is satisfied with $R_{2}(\eta)=2|P| \eta$, for all $\eta \geq 0$. From (57), we have for all $\alpha \in \mathbb{R}^{m}$ that

$$
\frac{d V(\alpha)}{d \alpha} \cdot A \alpha=2 P \alpha \cdot A \alpha=\alpha^{T}\left(P A+A^{T} P\right) \alpha=-|\alpha|^{2} .
$$

Therefore, (54) implies that for almost all $t \in \mathbb{R}_{+}$and $\gamma>0$ that

$$
\begin{aligned}
& \left.\frac{d V(\alpha)}{d \alpha}\right|_{\alpha=y_{\gamma}(t)} \cdot\left[A\left(y_{\gamma}(t)+q_{u}(t)\right)+B u(t)\right] g\left(\frac{\dot{u}(t)}{\gamma}\right) \\
& =-g\left(\frac{\dot{u}(t)}{\gamma}\right)\left|y_{\gamma}(t)\right|^{2} \leq-\frac{L M^{\lambda}}{2 \gamma^{\lambda}}\left|y_{\gamma}(t)\right|^{2},
\end{aligned}
$$

where $y_{\gamma}$ is defined in (39). Thus, (42) is satisfied with $\beta_{3}(\eta)=$ $\left(L M^{\lambda} / 2\right) \eta^{2}$, for all $\eta \geq 0$ and $R_{1}(\eta)=0$, for all $\eta \geq 0$.

Let $\delta_{1}$ be the zero function. Then (40) is verified. Take $\delta_{2}, \gamma_{*}$ arbitrary in $\mathbb{R}_{+}\left(\right.$say $\left.\delta_{2}=1, \gamma_{*}=1\right)$. Hence, all conditions of Lemma 20 are satisfied. Thus, it follows from Lemma 20 that there exist some $E, \gamma^{*}>0$ such that for all $\gamma>$ $\gamma^{*}$, the solution of (52) is global with $\left|x_{\gamma}(t)\right| \leq E$, for all $t \geq 0$. Moreover, the operator which maps $\left(u, x_{0}\right)$ to $x$ is consistent. In particular, we have $\left\|\sigma_{\gamma}-q_{u}\right\|_{\infty}=\left\|\sigma_{\gamma}+A^{-1} B u\right\|_{\infty} \rightarrow 0$ as $\gamma \rightarrow \infty$.
As a conclusion, the graphs $\left\{\left(\sigma_{\gamma}(t), u(t)\right), t \geq 0\right\}$ converge to the graph of the linear function $R: \mathbb{R} \rightarrow \mathbb{R}^{m}$, which is defined as $R(\alpha)=-A^{-1} B \alpha$, for all $\alpha \in \mathbb{R}$. This means that for $\lambda \in(0,1)$, the model (52) does not represent a hysteresis (see Remark 21).

Simulations. Take $m=1, B=1.0, A=-1.0$, and $x_{0}=0$. Let $g(\vartheta)=\sqrt{|\mathfrak{\vartheta}|}$, for all $\vartheta \in \mathbb{R}$, then $d_{+}^{\lambda} g(0)=d_{-}^{\lambda} g(0)=$ $\Gamma(3 / 2)=\Gamma(1 / 2) / 2=\sqrt{\pi} / 2>0$. Let $u \in W^{1, \infty}\left(\mathbb{R}_{+}, \mathbb{R}\right)$ be the function of period 2 such that $u(t)=t$, for all $t \in[0,1]$, and $u(t)=2-t$, for all $t \in[1,2]$. Then, we have $|\dot{u}(t)|=1$, for almost all $t \geq 0$. We also have $q_{u}=-A^{-1} B u=u$. Figure 2(a) shows that the graph $\left\{\left(\sigma_{\gamma}(t), u(t)\right), t \geq 0\right\}$ collapses into the identity function when $\gamma \rightarrow \infty$. This happens because of the fact that $q_{u}=u$ and Remark 21. Figure 2(b) shows that the sequence of functions $\sigma_{\gamma}$ converges uniformly to $q_{u}=u$ as $\gamma \rightarrow \infty$.

6.2. Class $\lambda=1$ Functions. In this subsection, we consider class $\lambda=1$ functions. (A function $g \in C^{0}\left(\mathbb{R}, \mathbb{R}^{r}\right)$ is of class $\lambda=1$ if $g(0)=\mathbf{0}$ and the limits $\lim _{\kappa \rightarrow 0+} g(\kappa) /|\kappa|$ and $\lim _{\kappa \rightarrow 0-} g(\kappa) /|\kappa|$ exist, are finite, and at least one of them is nonzero (see Section 5)). The main results of this subsection are given in Lemmas 23 and 27.

Lemma 23. Assume the following.

(1) The system (1)-(2) has a unique global solution.

(2) For the function $g$ in system (1)-(2), there exist $a_{1}, a_{2} \in$ $\mathbb{R}^{r}$ such that

$$
g(\vartheta)= \begin{cases}a_{1} \vartheta & \vartheta \geq 0 \\ a_{2} \vartheta & \vartheta<0\end{cases}
$$

Then, the sequence of functions $\sigma_{\gamma}$ of (5) is independent of $\gamma$ and the operator which maps $\left(u, x_{0}\right)$ to $x$ is consistent.

Proof. By condition (2), the right-hand side of (5) is independent of $\gamma$. Thus, the solution $\sigma_{\gamma}$ of (5) is independent of $\gamma$. Since $x_{u \circ s_{\gamma}} \circ \rho_{u}=\sigma_{\gamma}$, the function $x_{u \circ s_{\gamma}}$ is also independent of $\gamma$ (this is the so-called "rate-independent hysteresis") and hence consistency holds.

Example 24. Consider Bouc's hysteresis model [13] as follows:

$$
\dot{x}=-c|\dot{u}| x+\Phi^{\prime}(u) \dot{u},
$$

where $c>0, \Phi \in C^{1}(\mathbb{R}, \mathbb{R})$, input $u \in W^{1, \infty}\left(\mathbb{R}_{+}, \mathbb{R}\right)$, and $\Phi^{\prime}(u)=d \Phi(\alpha) /\left.d \alpha\right|_{\alpha=u}$.

The right-hand side of (62) is Lipschitz with respect to $x$. Thus, the system has a unique solution. Furthermore, we have

$$
\begin{aligned}
& \dot{x} \leq-\left|\Phi^{\prime}(u) \dot{u}\right|+\Phi^{\prime}(u) \dot{u} \leq 0, \quad \text { when } x \geq \frac{1}{c}\left|\Phi^{\prime}(u)\right|, \\
& \dot{x} \geq\left|\Phi^{\prime}(u) \dot{u}\right|+\Phi^{\prime}(u) \dot{u} \geq 0, \text { when } x \leq-\frac{1}{c}\left|\Phi^{\prime}(u)\right| .
\end{aligned}
$$




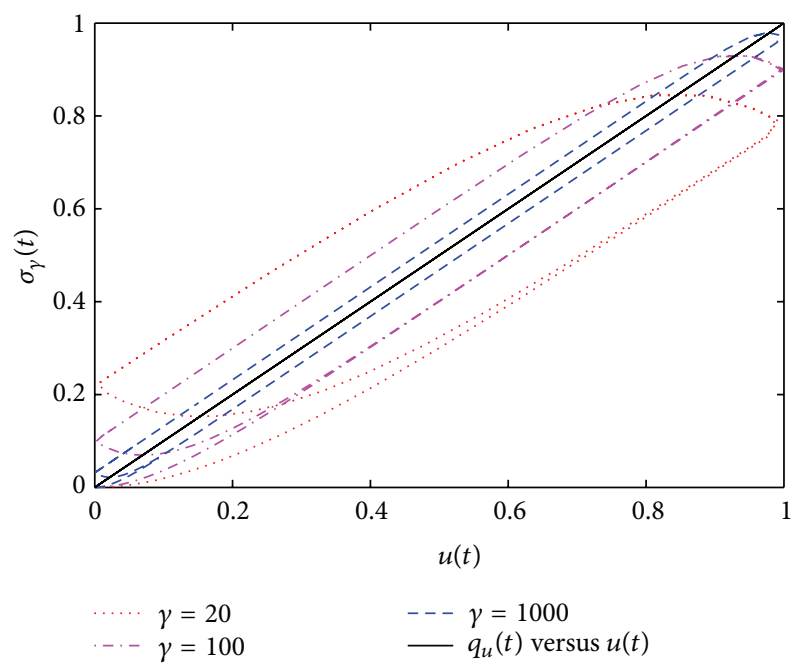

(a) $\sigma_{\gamma}(t)$ versus $u(t)$ for system (52)

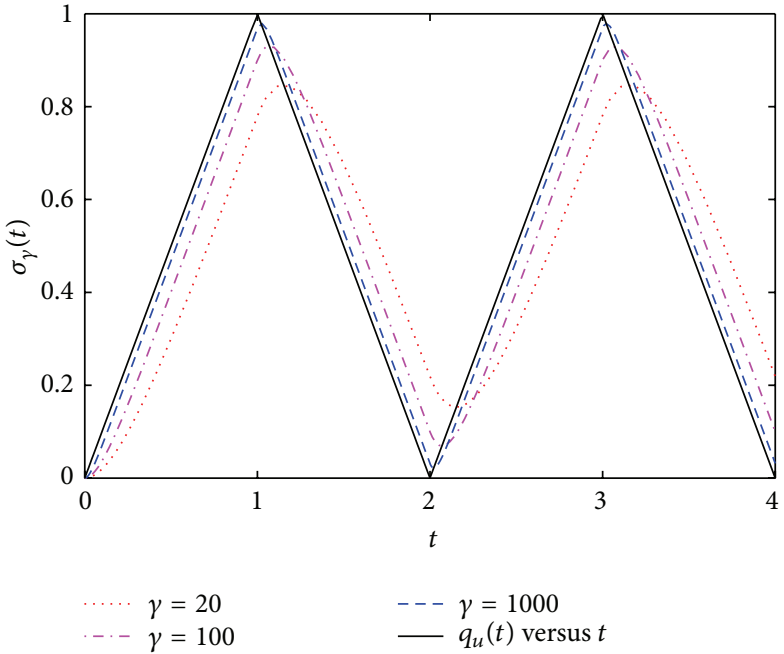

(b) $\sigma_{\gamma}(t)$ versus $t$ for system (52)

FIGURE 2: (a) $\sigma_{\gamma}(t)$ versus $u(t)$ for different values of $\gamma$. (b) $\sigma_{\gamma}(t)$ versus $t$ for different values of $\gamma$. In each plot, the function $q_{u}$ is the solid line.

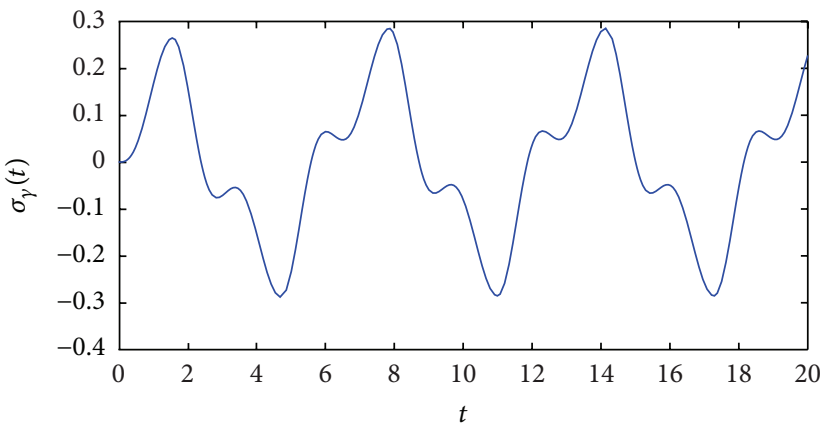

(a) $\sigma_{\gamma}(t)$ versus $t$

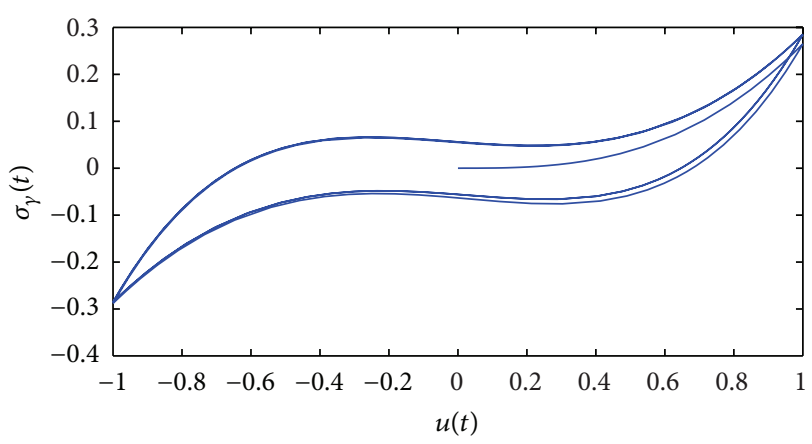

(b) $\sigma_{\gamma}(t)$ versus $u(t)$

Figure 3: (a) $\sigma_{\gamma}(t)$ versus $t$. (b) $\sigma_{\gamma}(t)$ versus $u(t)$ for Boucs hysteresis model (62).

Thus, $|x| \leq \max \left\{\left|\Phi^{\prime}(u)\right| / c,|x(0)|\right\}$, for each input $u \in$ $W^{1, \infty}\left(\mathbb{R}_{+}, \mathbb{R}\right)$ and each initial state $x(0) \in \mathbb{R}$. Since $u$ is bounded and $\Phi^{\prime}(u)$ is continuous, the solution $x$ of (62) is bounded and hence global. Hence, condition (1) in Lemma 23 is satisfied. Equation (62) can be written as

$$
\begin{aligned}
\dot{x} & =f(x, u) g(\dot{u}) \\
& =\left[-c x+\Phi^{\prime}(u) c x+\Phi^{\prime}(u)\right]\left[\begin{array}{c}
\max \{0, \dot{u}\} \\
\min \{0, \dot{u}\}
\end{array}\right] .
\end{aligned}
$$

Clearly, the function $g$ is of class $\lambda=1$ and satisfies condition (2) in Lemma 23. This fact implies that the operator which maps $(u, x(0))$ to $x$ is consistent and $\sigma_{\gamma}$ is independent of $\gamma$.

Simulations. Let $c=1, \Phi(\alpha)=\alpha^{3} / 3$, for all $\alpha \in \mathbb{R}, x(0)=0$, and input $u(t)=\sin (t)$, for all $t \geq 0$. The function $\sigma_{\gamma}$ is independent of $\gamma$ and is plotted in Figure 3(a). Furthermore, Figure 3(b) shows a rate-independent hysteresis behavior; that is graphs $\left\{\left(\sigma_{\gamma}(t), u(t)\right), t \geq 0\right\}$ are the same for different values of $\gamma$.
Proposition 25. Let $u \in W^{1, \infty}\left(\mathbb{R}_{+}, \mathbb{R}\right)$ be nonconstant. There exists a unique function $v_{u} \in L^{\infty}\left(I_{u}, \mathbb{R}\right)$ that is defined by $v_{u} \circ \rho_{u}=\dot{u}$. Moreover, $\left\|v_{u}\right\|_{\infty, I_{u}} \leq\|\dot{u}\|_{\infty}$. Assume that $\dot{u}$ is nonzero on a set $A \subseteq \mathbb{R}$ that satisfies $\mu\left(\rho_{u}(\mathbb{R} \backslash A)\right)=0$. Then, $v_{u}$ is nonzero almost everywhere.

Proof. Consider the left-derivative operator $\Delta_{-}$defined on $W^{1, \infty}\left(\mathbb{R}_{+}, \mathbb{R}\right)$ by $\left[\Delta_{-}(u)\right](t)=\lim _{\tau \uparrow t}((u(\tau)-u(t)) /(\tau-t))$. The operator $\Delta_{-}$is causal as $\left[\Delta_{-}(u)\right](t)$ depends only on values of $u(\tau)$ for $\tau \leq t$, and we have $\Delta_{-}(u)=\dot{u}$ almost every where as $u \in W^{1, \infty}\left(\mathbb{R}_{+}, \mathbb{R}\right)$ so that $\Delta_{-}(u) \in L^{\infty}\left(\mathbb{R}_{+}, \mathbb{R}\right)$. The operator $\Delta_{\text {_ }}$ satisfies Assumption 2. The first part of Proposition 25 follows immediately from Lemma 3 . Now, let $B=\left\{\varrho \in I_{u} / v_{u}(\varrho)=0\right\}$, then $B \subseteq \rho_{u}(\mathbb{R} \backslash A)$ which implies that $\mu(B)=0$.

Remark 26. Observe that if $\dot{u}$ is nonzero almost everywhere, then $\mu(\mathbb{R} \backslash A)=0$ so that by [26] we have $\mu\left(\rho_{u}(\mathbb{R} \backslash A)\right)=0$ as $\rho_{u}$ is absolutely continuous. An example in which $\dot{u}$ does not need to be nonzero almost everywhere, is when $u$ is constant on some interval, or on a finite number of intervals, or an 
infinite number of intervals such that this infinite number has measure zero (e.g., countable).

Lemma 27. Let $u \in W^{1, \infty}\left(\mathbb{R}_{+}, \mathbb{R}\right)$ be such that $\dot{u}$ is nonzero on a set $A \subseteq \mathbb{R}$ that satisfies $\mu\left(\rho_{u}(\mathbb{R} \backslash A)\right)=0$. Consider the semilinear Duhem model with $m=1$ and $\lambda=1$ as follows:

$$
\begin{gathered}
\dot{x}=(A x+B u+C) g(\dot{u}), \\
x(0)=x_{0},
\end{gathered}
$$

where $A=\left[\begin{array}{llll}a_{1} & a_{2} & \cdots & a_{r}\end{array}\right] \neq \mathbf{0}, B$, and $C$ are $1 \times r$ row vectors, state $x \in \mathbb{R}$ and function $g \in C^{0}\left(\mathbb{R}, \mathbb{R}^{r}\right)$ and of class $\lambda=1$, and nonconstant input $u \in W^{1, \infty}\left(\mathbb{R}_{+}, \mathbb{R}\right)$. Denote

$$
\begin{gathered}
\lim _{\kappa \rightarrow 0+} \frac{g(\kappa)}{|\kappa|}=G_{+}^{*}=\left[\begin{array}{c}
G_{+, 1}^{*} \\
G_{+, 2}^{*} \\
\vdots \\
G_{+, r}^{*}
\end{array}\right], \\
\lim _{\kappa \rightarrow 0-} \frac{g(\kappa)}{|\kappa|}=G_{-}^{*}=\left[\begin{array}{c}
G_{-, 1}^{*} \\
G_{-, 2}^{*} \\
\vdots \\
G_{-, r}^{*}
\end{array}\right] .
\end{gathered}
$$

For any $i \in\{1,2, \ldots, r\}$, assume that

$$
\begin{aligned}
& G_{+, i}^{*}, G_{-, i}^{*} \geq 0, \quad \text { whenever } a_{i}<0, \\
& G_{+, i}^{*}, G_{-, i}^{*} \leq 0, \quad \text { whenever } a_{i}>0 .
\end{aligned}
$$

Suppose that there exists some $i_{0} \in\{1,2, \ldots, r\}$, such that

$$
a_{i_{0}} \neq 0, \quad\left|G_{+, i_{0}}^{*}\right|+\left|G_{-, i_{0}}^{*}\right|>0 .
$$

Then,

(i) there exist E, $\gamma_{1}>0$ such that $\left\|x_{\gamma}\right\|_{\infty} \leq E$, for all $\gamma>$ $\gamma_{1}\left(x_{\gamma}\right.$ is the output of the system (65) when we use the input $u \circ s_{\gamma}$ instead of the input $u$ (see system (3)-(4))).

(ii) There exists a function $q_{u} \in W^{1, \infty}\left(\mathbb{R}_{+}, \mathbb{R}\right)$ such that $\left\|\sigma_{\gamma}-q_{u}\right\|_{1, \infty} \rightarrow 0$, as $\gamma \rightarrow \infty$ where $\|\cdot\|_{1, \infty}$ is the norm of the Banach space $W^{1, \infty}\left(\mathbb{R}_{+}, \mathbb{R}^{m}\right.$ ) (and hence the consistency of the system (65) is guaranteed with respect to input $u$ and initial condition $x_{0}$ ).

(iii) $q_{u}(0)=x_{0}$. Furthermore, for almost all $t \geq 0$, one has

$$
\dot{q}_{u}(t)=\left(A q_{u}(t)+B u(t)+C\right) g^{*}(\dot{u}(t)),
$$

where the function $g^{*} \in C^{0}(\mathbb{R}, \mathbb{R})$ is defined as in (9), that is;

$$
g^{*}(\vartheta)= \begin{cases}\vartheta G_{+}^{*} & \vartheta \geq 0 \\ -\vartheta G_{-}^{*} & \vartheta<0 .\end{cases}
$$

Proof. The semilinear Duhem model has a unique global solution due to the Lipschitz property of the right-hand-side [21, page 5].

For any $i \in\{1,2, \ldots, r\}$ and any $\kappa \in \mathbb{R} \backslash\{0\}$, let $G_{i}(\kappa)$ be the $i$ th component of the function $g(\kappa) /|\kappa|$. From (66), there exists some constant $\zeta>0$, such that for all $i \in\{1,2, \ldots, r\}$

$$
\begin{aligned}
G_{+, i}^{*}-\frac{\left|G_{+, i}^{*}\right|}{2} & <G_{i}(\kappa) \\
& <G_{+, i}^{*}+\frac{\left|G_{+, i}^{*}\right|}{2}, \quad \text { whenever } \kappa \in(0, \zeta), \\
G_{-, i}^{*}-\frac{\left|G_{-, i}^{*}\right|}{2} & <G_{i}(\kappa) \\
& <G_{-, i}^{*}+\frac{\left|G_{-, i}^{*}\right|}{2}, \quad \text { whenever } \kappa \in(-\zeta, 0) .
\end{aligned}
$$

Let $i \in\{1,2, \ldots, r\}$. We get from (67) and (71) that

$$
\begin{aligned}
a_{i} G_{i}(\kappa) & \leq-\max \left\{\frac{\left|a_{i}\right|\left|G_{+, i}^{*}\right|}{2}, \frac{\left|a_{i}\right|\left|G_{-, i}^{*}\right|}{2}\right\} \\
& =-M_{i}, \quad \text { whenever } \kappa \in(-\zeta, \zeta) \backslash\{0\}, a_{i} \neq 0 .
\end{aligned}
$$

For the case $a_{i}=0$, take $M_{i}=0$. Let $M=\sum_{i=1}^{r} M_{i}$. From (67) and (68), we get $M>0$. Thus, we get from (72) that

$$
A \frac{g(\kappa)}{|\kappa|}=\sum_{i=1}^{r} a_{i} G_{i}(\kappa) \leq-M<0, \quad \forall \kappa \in(-\zeta, \zeta) \backslash\{0\} .
$$

By Proposition 25, a function $v_{u} \in L^{\infty}\left(I_{u}, \mathbb{R}\right)$ can be defined almost every where as $v_{u} \circ \rho_{u}=\dot{u}$ with $v_{u} \neq \mathbf{0}$ almost every where The boundedness of $v_{u}$ implies that there exists $\gamma_{0}>0$ such that $v_{u}(\varrho) / \gamma \in(-\zeta, \zeta)$ for almost all $\varrho \in I_{u}$ and all $\gamma>\gamma_{0}$. Thus, we deduce from (73) that for all $\gamma>\gamma_{0}$,

$$
\gamma A \frac{g\left(v_{u}(\varrho) / \gamma\right)}{\left|v_{u}(\varrho)\right|} \leq-M, \quad \text { for almost all } \varrho \in I_{u} .
$$

Let $H: \mathbb{R} \rightarrow \mathbb{R}^{r}$ be a function such that

$$
H(\kappa)= \begin{cases}G_{+}^{*} & \kappa>0 \\ G_{-}^{*} & \kappa<0\end{cases}
$$

For any $\gamma>0$, define $\chi_{\gamma}: I_{u} \rightarrow \mathbb{R}^{r}$ as

$$
\chi_{\gamma}(\varrho)= \begin{cases}\gamma \frac{g\left(v_{u}(\varrho) / \gamma\right)}{\left|v_{u}(\varrho)\right|}-H\left(v_{u}(\varrho)\right) & v_{u}(\varrho) \neq 0, \\ 0 & \text { otherwise. }\end{cases}
$$

Since $v_{u} \in L^{\infty}\left(I_{u}, \mathbb{R}\right)$, relations (66) imply that

$$
\lim _{\gamma \rightarrow \infty}\left\|x_{\gamma}\right\|_{\infty}=0
$$

This result can be easily checked using the same techniques used in the proof of Proposition 9. Now, consider the system

$$
\begin{gathered}
\dot{h}=\left(A h+B \psi_{u}+C\right)\left(H \circ v_{u}\right), \\
h(0)=x_{0},
\end{gathered}
$$


where state $h \in \mathbb{R}$. The differential equation (78) verifies Carathéodory conditions with Lipschitz property with respect to $h$. Thus, system (78)-(79) has a unique and absolutely continuous local solution [21, page 4]. Consider the Lyapunov function $W=h^{2}$. We deduce from (78)-(79) that $W(0)=x_{0}^{2}$ and that

$$
\dot{W}=2 A\left(H \circ v_{u}\right) W+2\left(B \psi_{u}+C\right)\left(H \circ v_{u}\right) \sqrt{W} .
$$

We get from (67) and (68) that $A H\left(v_{u}(\varrho)\right) \leq$ $-\sum_{i=1}^{r} \max \left\{\left|a_{i}\right|\left|G_{+, i}^{*}\right|,\left|a_{i}\right|\left|G_{-, i}^{*}\right|\right\}=-2 M$ for almost all $\varrho \in I_{u}$. Thus, the boundedness of the function $\psi_{u}$ (see Lemma 1) along with (80) imply that $\dot{W} \leq-4 M W+D_{1} \sqrt{W}$, for some $D_{1}>0$. This leads to $\dot{W} \leq 0$, whenever $W \geq\left(D_{1} / 4 M\right)^{2}$. Therefore, Lemma 17 and the fact that $W(0)=x_{0}^{2}$ imply that $W \leq \max \left\{x_{0}^{2},\left(D_{1} / 4 M\right)^{2}\right\}$ which means that the solution of the system (78)-(79) is bounded and hence is global (i.e., is defined on $I_{u}$ ). On the other hand, the relation $\sigma_{\gamma}=x_{u \circ s_{\gamma}} \circ \rho_{u}$ implies that $\dot{\sigma}_{\gamma}=|\dot{u}| \dot{x}_{u \circ s_{\gamma}} \circ \rho_{u}$. Thus, we obtain from systems (65) and (5) and the relations $v_{u} \circ \rho_{u}=\dot{u}$, and $\psi_{u} \circ \rho_{u}=u$, that $x_{u \circ s_{\gamma}}(0)=x_{0}$ and that

$$
\dot{x}_{u \circ s_{\gamma}}(\varrho)=\gamma\left(A x_{u \circ s_{\gamma}}(\varrho)+B \psi_{u}(\varrho)+C\right) \frac{g\left(v_{u}(\varrho) / \gamma\right)}{\left|v_{u}(\varrho)\right|}
$$

for all $\gamma>0$, for almost all $\varrho \in\left[0, \tau_{\gamma}\right) \subseteq I_{u}$, where $\left[0, \tau_{\gamma}\right)$ is the maximal interval of existence [21, page 4]. For any $\gamma>0$, let $y_{\gamma}: I_{u} \rightarrow \mathbb{R}$ be defined as $y_{\gamma}=x_{u \circ s_{\gamma}}-h$. Since $x_{u \circ s_{\gamma}}(0)=$ $h(0)=x_{0}$, the system (81) can be written for all $\gamma>0$, for almost all $\varrho \in\left[0, \tau_{\gamma}\right)$ as

$$
\begin{gathered}
\dot{y}_{\gamma}(\varrho)=\gamma A \frac{g\left(v_{u}(\varrho) / \gamma\right)}{\left|v_{u}(\varrho)\right|} y_{\gamma}(\varrho) \\
+\left(A h(\varrho)+B \psi_{u}(\varrho)+C\right) \chi_{\gamma}(\varrho), \\
y_{\gamma}(0)=0 .
\end{gathered}
$$

For any $\gamma>0$, consider the Lyapunov function $V_{\gamma}:\left[0, \tau_{\gamma}\right) \rightarrow$ $\mathbb{R}_{+}$with $V_{\gamma}(\varrho)=y_{\gamma}^{2}(\varrho)$, for all $\varrho \in\left[0, \tau_{\gamma}\right)$. By $(82)$ and the boundedness of both $\psi_{u}$ and the solution of (78)-(79), there exists some $D_{2}>0$ such that for almost all $\varrho \in\left[0, \tau_{\gamma}\right)$ and all $\gamma>\gamma_{0}$

$$
\dot{V}_{\gamma}(\varrho) \leq 2 \gamma A \frac{g\left(v_{u}(\varrho) / \gamma\right)}{\left|v_{u}(\varrho)\right|} V_{\gamma}(\varrho)+D_{2}\left\|x_{\gamma}\right\|_{\infty} \sqrt{V_{\gamma}(\varrho)} .
$$

Thus, we get from (74) that

$$
\begin{array}{r}
\dot{V}_{\gamma}(\varrho) \leq 0, \quad \text { for almost all } \varrho \in\left[0, \tau_{\gamma}\right), \forall \gamma>\gamma_{0}, \\
\text { that satisfy } V_{\gamma}(\varrho)>\left(\frac{D_{2}\left\|\chi_{\gamma}\right\|_{\infty}}{2 M}\right)^{2} .
\end{array}
$$

Therefore, Lemma 17 and the fact that $V_{\gamma}(0)=0$ imply that $V_{\gamma}(\varrho) \leq\left(D_{2}\left\|\chi_{\gamma}\right\|_{\infty} / 2 M\right)^{2}$ for all $\gamma>\gamma_{0}$ and almost all $\varrho \in$ $\left[0, \tau_{\gamma}\right)$, and hence we obtain, for almost all $\varrho \in\left[0, \tau_{\gamma}\right)$ that

$$
\left|y_{\gamma}(\varrho)\right|=\left|x_{u \circ s_{\gamma}}(\varrho)-h(\varrho)\right| \leq \frac{D_{2}}{2 M}\left\|\chi_{\gamma}\right\|_{\infty}, \quad \forall \gamma>\gamma_{0},
$$

which implies that $\left[0, \tau_{\gamma}\right)=I_{u}$, for all $\gamma>\gamma_{0}$ and (77) implies that

$$
\lim _{\gamma \rightarrow \infty}\left\|x_{u \circ s_{\gamma}}-h\right\|_{\infty, I_{u}}=0
$$

On the other hand, the continuity of $x_{\gamma}$ implies that $\left\|x_{u \circ s_{\gamma}}\right\|_{\infty, I_{u}}=\left\|x_{\gamma}\right\|_{\infty}$, for all $\gamma>\gamma_{0}$ (see Lemma 3). Thus, there exists some $E>0$ and $\gamma_{1}>\gamma_{0}$ with

$$
\left\|x_{u^{\circ} s_{\gamma}}\right\|_{\infty}=\left\|x_{\gamma}\right\|_{\infty} \leq E, \quad \forall \gamma>\gamma_{1} \text {. }
$$

Moreover, we get from (78) and (81) for all $\gamma>\gamma_{1}$ that

$$
\begin{aligned}
\dot{x}_{u \circ s_{\gamma}}-\dot{h}= & \left(A x_{u \circ s_{\gamma}}+B \psi_{u}+C\right) \chi_{\gamma} \\
& +A\left(x_{u \circ s_{\gamma}}-h\right)\left(H \circ v_{u}\right) .
\end{aligned}
$$

Thus, by the boundedness of functions $\dot{u}$ and $\psi_{u}$, and the relation (88), there exist positive constants $D_{3}$ and $D_{4}$ independent of $\gamma$, such that

$$
\left|\dot{x}_{u \circ s_{\gamma}}-\dot{h}\right| \leq D_{3}\left\|x_{\gamma}\right\|_{\infty}+D_{4}\left\|x_{u \circ s_{\gamma}}-h\right\|_{\infty}, \quad \forall \gamma>\gamma_{1} \text {, }
$$

which means that $x_{u \circ s_{\gamma}}$ converges to $h$ in $W^{1, \infty}\left(I_{u}, \mathbb{R}\right)$ as $\gamma \rightarrow$ $\infty$ because of (77) and (87). Define $q_{u} \in C^{0}\left(\mathbb{R}_{+}, \mathbb{R}\right)$ as $q_{u}=$ $h \circ \rho_{u}$. Since for all $\dot{q}_{u}=|\dot{u}| \dot{h} \circ \rho_{u}$, relations (78)-(79) imply for all $t \geq 0$ that

$$
q_{u}(t)=x_{0}+\int_{0}^{t}\left(A q_{u}(\tau)+B u(\tau)+C\right) g^{*}(\dot{u}(\tau)) d \tau .
$$

Moreover, using the relation $\sigma_{\gamma}=x_{u \circ s_{\gamma}} \circ \rho_{u}$, it can be easily verified that $\lim _{\gamma \rightarrow \infty}\left\|\sigma_{\gamma}-q_{u}\right\|_{1, \infty}=0$.

Remark 28. In Lemma 27, the sequence of functions $\sigma_{\gamma}$ converges in $W^{1, \infty}\left(\mathbb{R}_{+}, \mathbb{R}\right)$ as $\gamma \rightarrow \infty$. This result is stronger than the one obtained in Lemma 20, where the convergence is only in $L^{\infty}\left(\mathbb{R}_{+}, \mathbb{R}\right)$. An application to this stronger convergence is given in the following example.

Example 29. The LuGre model is described by [10] as follows:

$$
\begin{aligned}
& \dot{x}=\dot{u}-\theta \frac{|\dot{u}|}{\zeta(\dot{u})} x=\left[\begin{array}{ll}
1 & x
\end{array}\right]\left[\begin{array}{c}
\dot{u} \\
-\theta \frac{|\dot{u}|}{\zeta(\dot{u})}
\end{array}\right]=f(x) g(\dot{u}), \\
& x(0)=x_{0}, \\
& F=\theta x+c_{1} \dot{x}+c_{2} \dot{u},
\end{aligned}
$$

where parameters $\theta, c_{1}$, and $c_{2}>0$ are, respectively, the stiffness, damping, and viscous friction coefficients, $x \in \mathbb{R}$ is the average deflection of the bristles, $x_{0} \in \mathbb{R}$ is the initial state, $u \in W^{1, \infty}\left(\mathbb{R}_{+}, \mathbb{R}\right)$ is the relative displacement and is the input of the system, and $F$ is the friction force and is the output of the system. The function $\zeta: \mathbb{R} \rightarrow \mathbb{R}$ is defined as

$$
\zeta(\alpha)=F_{C}+\left(F_{S}-F_{C}\right) e^{-\left|\alpha / v_{s}\right|}, \quad \forall \alpha \in \mathbb{R}
$$


where $F_{C}>0$ is the Coulomb friction force, $F_{S}>0$ is the stiction force, and $v_{s}>0$ is the Stribeck velocity.

The LuGre model can be written in the form of the system (65) with $A=\left[\begin{array}{ll}a_{1} & a_{2}\end{array}\right]=\left[\begin{array}{ll}0 & 1\end{array}\right], B=\left[\begin{array}{ll}0 & 0\end{array}\right]$, and $C=\left[\begin{array}{ll}1 & 0\end{array}\right]$. We have

$$
\begin{gathered}
\lim _{\kappa \rightarrow 0+} \frac{g(\kappa)}{|\kappa|}=G_{+}^{*}=\left[\begin{array}{l}
G_{+, 1}^{*} \\
G_{+, 2}^{*}
\end{array}\right]=\left[\begin{array}{c}
1 \\
\frac{-\theta}{F_{S}}
\end{array}\right], \\
\lim _{\kappa \rightarrow 0-} \frac{g(\kappa)}{|\kappa|}=G_{-}^{*}=\left[\begin{array}{l}
G_{-, 1}^{*} \\
G_{-, 2}^{*}
\end{array}\right]=\left[\begin{array}{c}
-1 \\
\frac{-\theta}{F_{S}}
\end{array}\right] .
\end{gathered}
$$

Clearly, conditions (67) and (68) are satisfied. Thus, Lemma 27 implies that $\left\|\sigma_{\gamma}-q_{u}\right\|_{1, \infty} \rightarrow 0$, as $\gamma \rightarrow \infty$, where the functions $\sigma_{\gamma}: \mathbb{R}_{+} \rightarrow \mathbb{R}$ and $q_{u} \in W^{1, \infty}\left(\mathbb{R}_{+}, \mathbb{R}\right)$ are defined for all $t \geq 0$ as

$$
\begin{gathered}
\sigma_{\gamma}(t)=x_{0}+\int_{0}^{t}\left(\dot{u}(\tau)-\frac{\theta|\dot{u}(\tau)|}{\zeta(\dot{u}(\tau) / \gamma)} q_{u}(\tau)\right) d \tau, \\
q_{u}(t)=x_{0}+\int_{0}^{t}\left(\dot{u}(\tau)-\frac{\theta|\dot{u}(\tau)|}{F_{S}} q_{u}(\tau)\right) d \tau .
\end{gathered}
$$

Also, there exist some $E, \gamma_{1}>0$ such that for all $\gamma>\gamma_{1}$, the solution of (92) is global with $\left\|x_{\gamma}\right\|_{\infty} \leq E$.

Now, the following analysis is not a part of Lemma 27, but it follows straightforwardly from it.

Let $F_{\gamma}$ be the output of the system when we use the input $u \circ s_{\gamma}$ instead of $u$. We obtain from (93) for almost all $t \geq 0$ that

$$
F_{\gamma}(t)=\theta x_{\gamma}(t)+c_{1} \dot{x}_{\gamma}(t)+\frac{c_{2}}{\gamma} \dot{u} \circ s_{\gamma}(t), \quad \forall \gamma>0,
$$

which leads to

$$
\sigma_{\gamma, F}(t)=\theta \sigma_{\gamma}(t)+c_{1} \frac{\dot{\sigma}_{\gamma}(t)}{\gamma}+\frac{c_{2}}{\gamma} \dot{u}(t), \quad \forall \gamma>0,
$$

where $\sigma_{\gamma, F}: \mathbb{R}_{+} \rightarrow \mathbb{R}^{m}$ is defined as $\sigma_{\gamma, F}(t)=F_{\gamma}(\gamma t)$, for all $\gamma>0$ and for all $t \geq 0$.

Since $\left\|\sigma_{\gamma}-q_{u}\right\|_{1, \infty} \rightarrow 0$, as $\gamma \rightarrow \infty$, we have $\lim _{\gamma \rightarrow \infty}\left\|\sigma_{\gamma}-q_{u}\right\|_{\infty}=0$ and $\lim _{\gamma \rightarrow \infty}\left\|\dot{\sigma}_{\gamma}-\dot{q}_{u}\right\|_{\infty}=0$. Thus, we get from the boundedness of $\dot{u}$ and (99) that

$$
\lim _{\gamma \rightarrow \infty}\left\|\sigma_{\gamma, F}(t)-\theta q_{u}\right\|_{\infty}=0
$$

which means that the operator which maps input $u$ and initial state $x_{0}$ to output $F$ is consistent.

The conclusion of the analysis is that the hysteresis loop of the LuGre model is $\left\{\left(\theta \dot{q}_{u}(t), u(t)\right), t \geq 0\right\}$, where $q_{u}$ is given in (97). Observe that this conclusion has been obtained due to the convergence of $\sigma_{\gamma}$ in $W^{1, \infty}\left(\mathbb{R}_{+}, \mathbb{R}\right)$ (see Remark 28).

Simulations. Take $\theta=4 \mathrm{~N} / \mathrm{m}, v_{s}=0.001 \mathrm{~m} / \mathrm{s}, F_{S}=3 \mathrm{~N}$, $F_{C}=1 \mathrm{~N}, c_{1}=1 \mathrm{~N} \cdot \mathrm{s} / \mathrm{m}, c_{2}=1 \mathrm{~N} \cdot \mathrm{s} / \mathrm{m}$, and $x(0)=0$ $\mathrm{N}$. Let $u \in W^{1, \infty}\left(\mathbb{R}_{+}, \mathbb{R}\right)$ be a function of period 2 that is measured in meters such that $u(t)=t$, for all $t \in[0,1] \mathrm{s}$, and $u(t)=2-t$, for all $t \in[1,2] \mathrm{s}$. Figure 4(a) shows the uniform convergence of $\sigma_{\gamma, F}$ to $\theta q_{u}$ as $\gamma \rightarrow \infty$. Figure 4(b) shows that the graphs $\left\{\left(\sigma_{\gamma}(t), u(t)\right), t \geq 0\right\}$ converge to the hysteresis loop $\left\{\left(\theta q_{u}(t), u(t)\right), t \geq 0\right\}$. Figures $4(\mathrm{c})$ and $4(\mathrm{~d})$ show that the sequence of functions $\sigma_{\gamma}$ and $\dot{\sigma}_{\gamma}$ converges uniformly to $q_{u}$ and $\dot{q}_{u}$ as $\gamma \rightarrow \infty$. Figures 4(e) and 4(f) give the graphs of the functions $q_{u}$ and $\dot{q}_{u}$.

\section{Conclusion}

This paper presented a classification of the possible Duhem models in terms of their consistency with the hysteresis behavior. Three classes of models have been considered in relation with the range of a parameter $\lambda$. For $\lambda>1$, it has been shown that the corresponding generalized Duhem model does not represent a hysteresis behavior. For $0<\lambda<$ 1 , it has been shown that the semilinear Duhem model is not compatible with a hysteresis behavior. In all other cases, necessary conditions and sufficient ones have been derived to insure the consistency of the Duhem model with the hysteresis property. Numerical simulations have been carried out to illustrate the obtained results.

\section{Appendices}

\section{A. Proof of Proposition 11}

Without loss of generality, assume that $r=1$. Definition 7 implies that there exists some $c_{0}>0$, such that

$$
\begin{gathered}
\left|\frac{g(\kappa)}{\kappa^{\lambda}}-\frac{d_{+}^{\lambda} g(0)}{\Gamma(1+\lambda)}\right|<1, \quad \forall \kappa \in\left(0, c_{0}\right), \\
\left|\frac{g(\kappa)}{(-\kappa)^{\lambda}}-\frac{d_{-}^{\lambda} g(0)}{\Gamma(1+\lambda)}\right|<1, \quad \forall \kappa \in\left(-c_{0}, 0\right) .
\end{gathered}
$$

Therefore, there exists some $d>0$ with

$$
|g(\kappa)| \leq d|\kappa|^{\lambda}, \quad \forall|\kappa|<c_{0} .
$$

Thus, the substitution $\kappa=\vartheta / \gamma$ implies that

$$
\begin{aligned}
&\left|\gamma^{\lambda} g\left(\frac{\vartheta}{\gamma}\right)\right| \leq d|\vartheta|^{\lambda}, \quad \forall \vartheta \in \mathbb{R}, \forall \gamma>0, \forall \gamma>\gamma_{1} \\
& \text { that satisfy }|\vartheta| / \gamma<c_{0} .
\end{aligned}
$$

Define $g_{1}: \mathbb{R} \rightarrow \mathbb{R}_{+}$, as

$$
g_{1}(\vartheta)= \begin{cases}\sup _{0 \leq s \leq \vartheta}|g(s)| & \vartheta>0, \\ 0 & \vartheta=0, \\ \sup _{\vartheta \leq s \leq 0}|g(s)| & \vartheta<0 .\end{cases}
$$




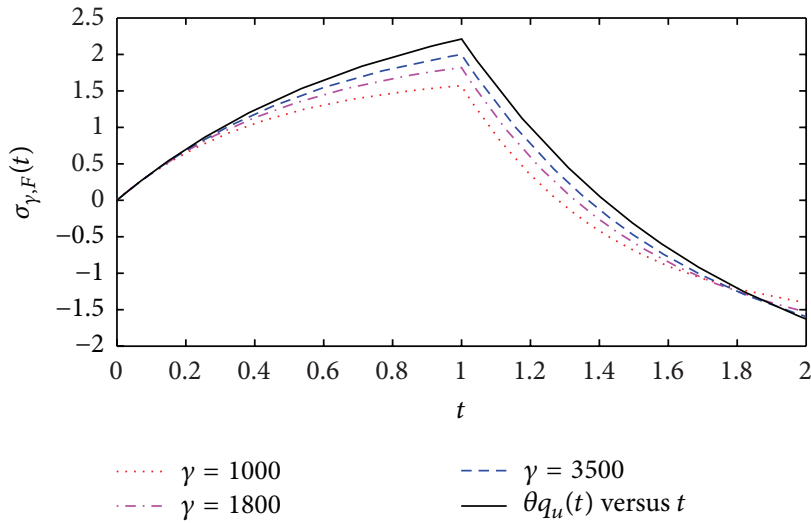

(a)

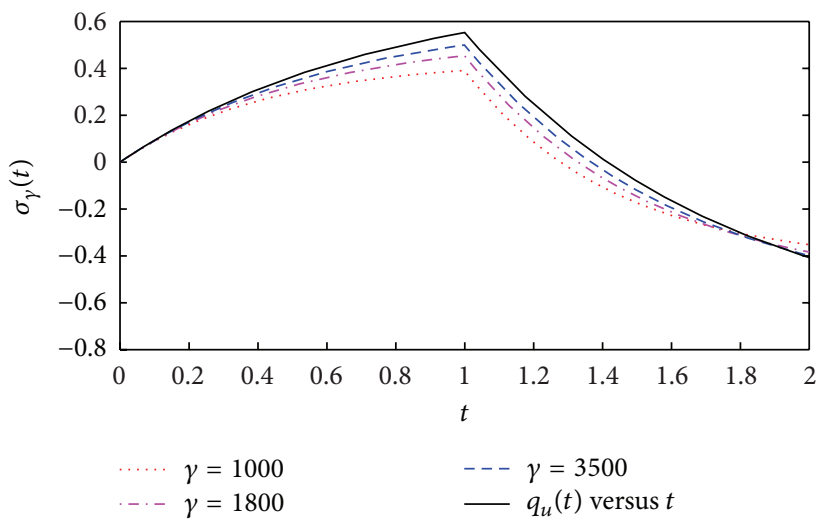

(c)

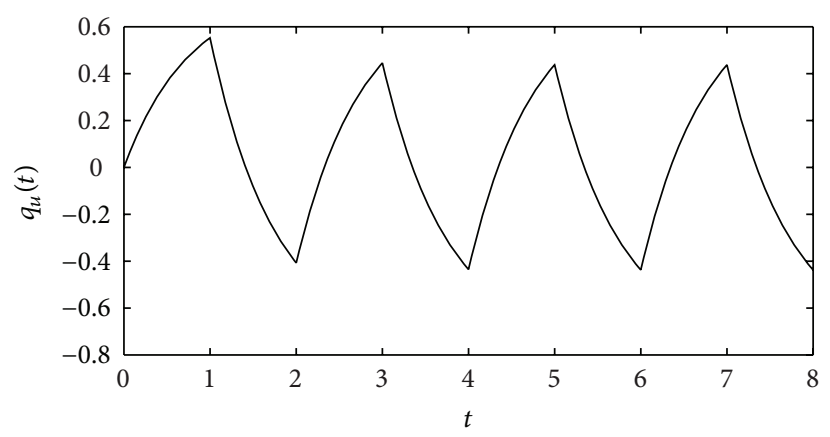

(e)

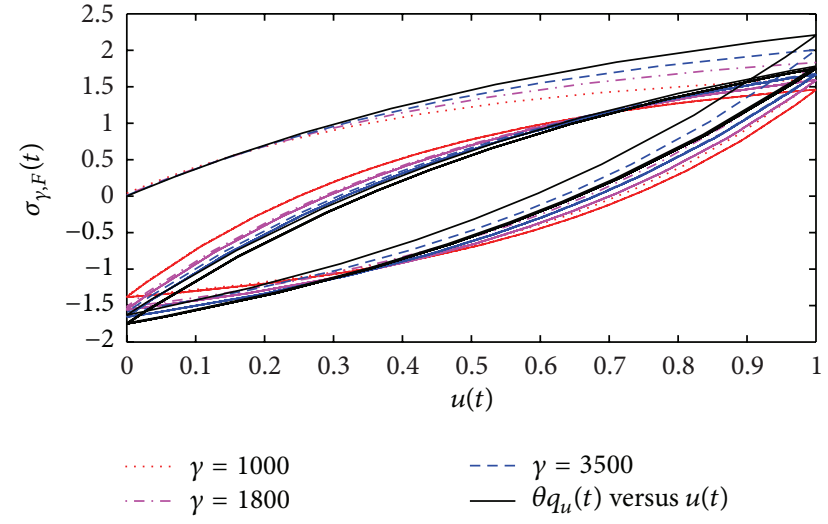

(b)

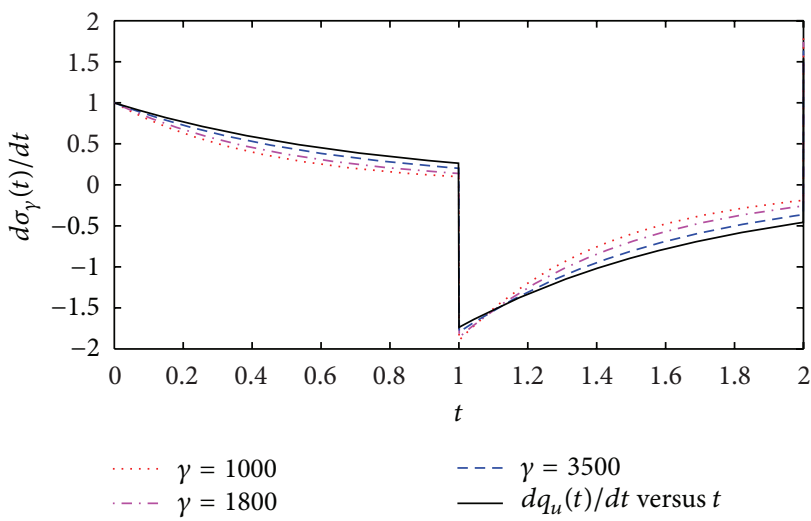

(d)

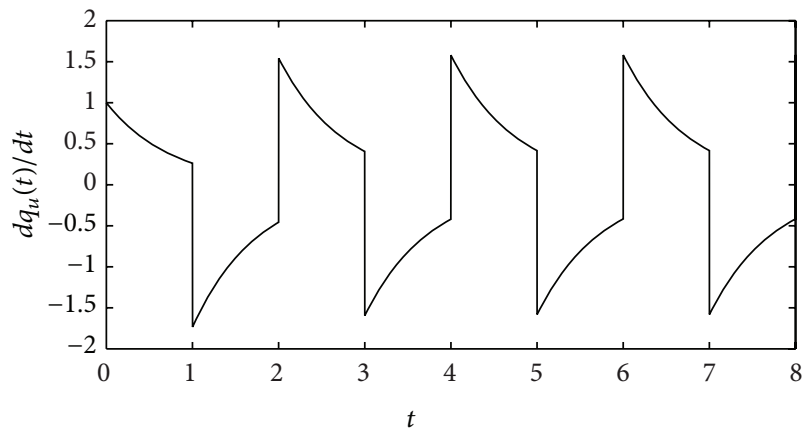

(f)

Figure 4: (a) $\sigma_{\gamma, F}(t)$ versus $t$. (b) $\sigma_{\gamma, F}(t)$ versus $u(t)$. (c) $\sigma_{\gamma}(t)$ versus $t$. (d) $\dot{\sigma}_{\gamma}(t)$ versus $t$. (e) $q_{u}(t)$ versus $t$. (f) $\dot{q}_{u}(t)$ versus $t$. The plots are for system (92) of Example 29. In each plot, $q_{u}$ and $\dot{q}_{u}$ are the solid black lines.

The function $g_{1}$ is continuous because $g$ is continuous. Moreover, we have $|g(\vartheta)| \leq g_{1}(\vartheta)$, for all $\vartheta \in \mathbb{R}$. The function $g_{1}$ is nondecreasing on $[0, \infty)$ and nonincreasing on $(-\infty, 0]$. This implies that

$$
\begin{aligned}
\left|\gamma^{\lambda} g\left(\frac{\vartheta}{\gamma}\right)\right| \leq \frac{|\vartheta|^{\lambda}}{c_{0}^{\lambda}} g_{1}(\vartheta), \quad \forall \vartheta \in \mathbb{R}, \forall \gamma>1 \\
\text { that satisfy } \frac{|\vartheta|}{\gamma} \geq c_{0} .
\end{aligned}
$$

Thus, we get from (A.3) for all $\vartheta \in \mathbb{R}$ and all $\gamma>1$ that

$$
\left|\gamma^{\lambda} g\left(\frac{\mathcal{\vartheta}}{\gamma}\right)\right| \leq \max \left\{d|\vartheta|^{\lambda}, \frac{|\mathcal{\vartheta}|^{\lambda}}{c_{0}^{\lambda}} g_{1}(\vartheta)\right\}=g_{0}(\vartheta),
$$

which completes the proof.

\section{B. Proof of Lemma 17}

We discuss two cases, Case $1 z(0) \leq z_{1}$ and Case $2 z_{1}<$ $z(0)<z_{2}$. 
Case $1\left(z(0) \leq z_{1}\right)$. The objective of what follows is to prove that for all $t \in[0, \omega)$ we have $z(t) \leq z_{1}$. To this end, assume that $\exists t_{1} \in(0, \omega)$, such that $z\left(t_{1}\right)>z_{1}$. Put $C=\{\tau \in$ $\left.(0, \omega) / z(t) \leq z_{1}, \forall t \in[0, \tau]\right\}$. The set $C$ is nonempty because $0 \in C$. Define $t_{2}:=\operatorname{Sup} C$; then there exists a real sequence $\left\{\tau_{n} \in C\right\}_{n=1}^{\infty}$ such that $\lim _{n \rightarrow \infty} \tau_{n}=t_{2}$. By the continuity of $z$, we have $z\left(t_{2}\right)=\lim _{n \rightarrow \infty} z\left(\tau_{n}\right) \leq z_{1}$. This fact implies that $t_{2} \in C$ leading to $t_{2}<t_{1}$. Also, there exists a real sequence $\left\{\tau_{n}^{\prime}>t_{2}\right\}_{n=1}^{\infty}$ such that $z\left(\tau_{n}^{\prime}\right)>z_{1}$, for all $n \in \mathbb{N}$ and $\lim _{n \rightarrow \infty} \tau_{n}^{\prime}=t_{2}$. Since $z$ is continuous, we get $z\left(t_{2}\right) \geq z_{1}$ which leads to $z\left(t_{2}\right)=z_{1}$. Let $D=\left\{t \in\left[t_{2}, t_{1}\right] / z(t)=z_{1}\right\}$. The set $D$ is nonempty since $t_{2} \in D$. Define $t_{3}:=\operatorname{Sup} D$; then using a similar argument as above we get $t_{3} \in D$ which implies that $z\left(t_{3}\right)=z_{1}$ and $t_{3}<t_{1}$.

Claim 1. $z(t)>z_{1}$, for all $t_{3}<t \leq t_{1}$.

Proof. Assume that $\exists t_{4} \in\left(t_{3}, t_{1}\right]$ such that $z\left(t_{4}\right) \leq z_{1}$. By definition of $t_{3}$, we get $z\left(t_{4}\right)<z_{1}$. Since $z$ is continuous and $z\left(t_{4}\right)<z_{1}<z\left(t_{1}\right)$, we can use the intermediate value theorem to find $t_{5} \in\left(t_{4}, t_{1}\right)$ such that $z\left(t_{5}\right)=z_{1}$ which implies that $t_{5} \in D$ and $t_{5}>t_{3}$ which is a contradiction.

Let $E=\left\{t \in\left[t_{3}, t_{1}\right] / z(t)=z_{2}\right\}$. We consider the following subcases $E=\emptyset$ and $E \neq \emptyset$. If $E=\emptyset$, then $z\left(t_{1}\right)<z_{2}$ and hence Claim 1 and (32) imply that

$$
\dot{z}(t) \leq 0, \quad \text { for almost all } t \in\left(t_{3}, t_{1}\right] .
$$

Thus, the absolute continuity of the function $z$ implies that $\int_{t_{3}}^{t_{1}} \dot{z}(t) d t=z\left(t_{1}\right)-z\left(t_{3}\right) \leq 0$ which contradicts the fact that $z\left(t_{3}\right)=z_{1}<z\left(t_{1}\right)$. Now, if $E \neq \emptyset$, let $t_{6}=\operatorname{Inf} E$. It can be shown that $t_{6} \in E$ and $t_{3}<t_{6}$. Thus, Claim 1 and (32) give

$$
\dot{z}(t) \leq 0, \quad \text { for almost all } t \in\left(t_{3}, t_{6}\right),
$$

which also contradicts the facts that $z$ is absolutely continuous on each compact interval and $z\left(t_{3}\right)=z_{1}<z_{2}=z\left(t_{6}\right)$.

We have, thus, proved that in Case 1 ,

$$
z(t) \leq z_{1}, \quad \forall t \in[0, \omega), \text { whenever } z(0) \leq z_{1} .
$$

Case $2\left(z_{1}<z(0)<z_{2}\right)$. Assume that $\exists t_{1} \in(0, \omega)$ such that $z\left(t_{1}\right)=z_{1}$. Let $t_{2}>0$ be the smallest real number such that $z\left(t_{2}\right)=z_{1}$ (it exists due to the continuity of $z$ ). Then, seeing $t_{2}$ as an initial time, and $z_{1}$ as an initial condition, it follows from Case 1 that for all $t \geq t_{2}$, we have $z(t) \leq z_{1}$. So, we have to just analyze what happens in the interval $\left[0, t_{2}\right]$ and discuss the case for all $t \in[0, \omega), z(t)>z_{1}$. The analysis of both situations is the same so that we focus on the case for all $t \in[0, \omega), z(t)>z_{1}$.

Assume that $\exists t_{3} \in(0, \omega)$ such that $z\left(t_{3}\right)=z_{2}$. Let $t_{4}>0$ be the smallest real number such that $z\left(t_{4}\right)=z_{2}$ (it exists due to the continuity of $z$ ). Then, for all $t \in\left[0, t_{4}\right)$, we have $z_{1}<z(t)<z_{2}$ which implies that for almost all $t \in\left[0, t_{4}\right)$ we have $\dot{z}(t) \leq 0$. Since $z$ is absolutely continuous, it follows that $\int_{0}^{t_{4}} \dot{z}(t) d t=z\left(t_{4}\right)-z(0)=z_{2}-z(0) \leq 0$. This contradicts the fact that $z_{2}>z(0)$ which means that for all $t \geq 0, z(t)<z_{2}$. Since for all $t \in[0, \omega), z(t)>z_{1}$, it follows that for all $t \in$
$[0, \omega), \dot{z}(t) \leq 0$ so that for all $t \in[0, \omega), \int_{0}^{t} \dot{z}(\tau) d \tau=z(t)-$ $z(0) \leq 0$.

As a conclusion, we have proved that in Case 2, for all $t \in$ $[0, \omega), z(t) \leq z(0)$.

\section{Acknowledgment}

Supported by Grant DPI2011-25822 of the Spanish Ministry of Economy and Competitiveness.

\section{References}

[1] A. Visintin, Differential Models of Hysteresis, Springer, Berlin, Germany, 1994.

[2] M. Brokate and J. Sprekels, Hysteresis and Phase Transitions, Springer, New York, NY, USA, 1996.

[3] M. A. Krasnosel'skii and A. V. Pokrovskii, Systems with Hysteresis, Springer, Berlin, Germany, 1989.

[4] F. Ikhouane and J. Rodellar, Systems with Hysteresis: Analysis, Identification and Control Using the Bouc-Wen Model, Wiley, Chichester, UK, 2007.

[5] I. Mayergoyz, Mathematical Models of Hysteresis, Elsevier Series in Electromagnetism, New York, NY, USA, 2003.

[6] P. Duhem, "Die dauernden aenderungen und die thermodynamik," Zeitschrift für Physikalische Chemie, vol. 22, pp. 543589, 1897.

[7] A. K. Padthe, B. Drincic, J. Oh, D. D. Rizos, S. D. Fassois, and D. S. Bernstein, "Duhem modeling of friction-induced hysteresis," IEEE Control Systems Magazine, vol. 28, no. 5, pp. 90-107, 2008.

[8] J. W. Macki, P. Nistri, and P. Zecca, "Mathematical models for hysteresis," SIAM Review, vol. 35, no. 1, pp. 94-123, 1993.

[9] J. Oh and D. S. Bernstein, "Semilinear Duhem model for rateindependent and rate-dependent hysteresis," IEEE Transactions on Automatic Control, vol. 50, no. 5, pp. 631-645, 2005.

[10] C. Canudas de Wit, H. Olsson, K. J. Astrom, and P. Lischinsky, "A new model for control of systems with friction," IEEE Transactions on Automatic Control, vol. 40, no. 3, pp. 419-425, 1995.

[11] P. R. Dahl, “A solid friction model,” Tech. Rep. TOR-0158 (310718), The Aerospace Corporation, El Segundo, Calif, USA, 1968.

[12] Y. K. Wen, "Method for random vibration of hysteretic systems," Journal of the Engineering Mechanics, vol. 102, no. 2, pp. 249263, 1976.

[13] R. Bouc, "Modèle mathématique d'hystérésis," Acustica, vol. 24, pp. 16-25, 1971.

[14] M. L. Hodgdon, "Applications of a theory of ferro-magnetic hysteresis," IEEE Transactions on Magnetics, vol. 24, no. 1, pp. 218-221, 1988.

[15] M. L. Hodgdon, "Mathematical theory and calculations of magnetic hysteresis curves," IEEE Transactions on Magnetics, vol. 24, no. 6, pp. 3120-3122, 1988.

[16] A. Rodríguez, N. Iwata, F. Ikhouane, and J. Rodellar, "Modeling and identification of a large-scale magnetorheological fluid damper," Smart Material and Structures, vol. 18, pp. 374-379, 2009.

[17] B. Jayawardhana, R. Ouyang, and V. Andrieu, "Stability of systems with the Duhem hysteresis operator: the dissipativity approach," Automatica, vol. 48, no. 10, pp. 2657-2662, 2012.

[18] R. Ouyang, V. Andrieu, and B. Jayawardhana, "On the characterization of the Duhem hysteresis operator with clockwise 
input-output dynamics," Systems \& Control Letters, vol. 62, no. 3, pp. 286-293, 2013.

[19] F. Ikhouane, "Characterization of hysteresis processes," Mathematics of Control, Signals, and Systems, vol. 25, no. 3, pp. 291-310, 2013.

[20] R. A. Adams and J. J. F. Fournier, Sobolev Spaces, Elsevier, 2003.

[21] A. F. Filippov, Differential Equations with Discon-Tinuous RightHand Sides, Kluwer Academic Publishers, 1988.

[22] F. B. Adda and J. Cresson, "About non-differentiable functions," Journal of Mathematical Analysis and Applications, vol. 263, no. 2, pp. 721-737, 2001.

[23] W. Rudin, Real and Complex Analysis, McGraw-Hill, 3rd edition, 1986.

[24] D. S. Bernstein, "Ivory ghost [ask the experts]," IEEE Control Systems, vol. 27, no. 5, pp. 16-17, 2007.

[25] H. K. Khalil, Nonlinear Systems, Prentice Hall, Upper Saddle River, NJ, USA, 3rd edition, 2002.

[26] D. E. Varberg, "On absolutely continuous functions," The American Mathematical Monthly, vol. 72, no. 8, pp. 831-841, 1965. 


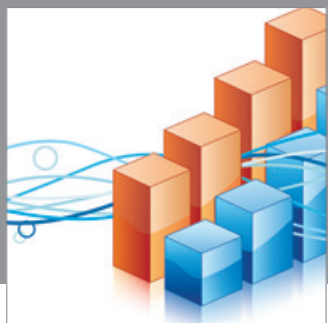

Advances in

Operations Research

mansans

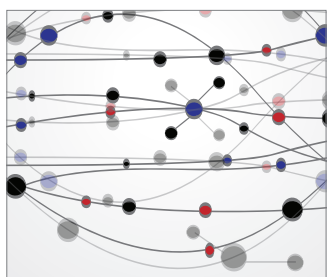

The Scientific World Journal
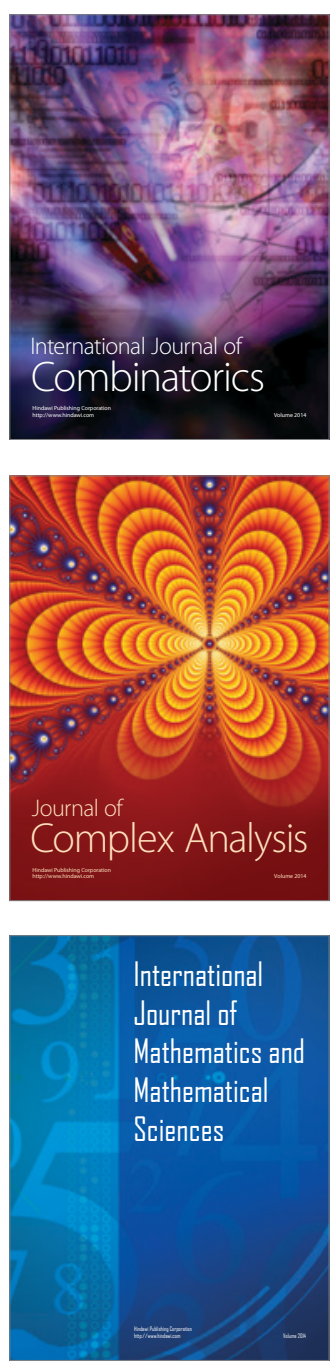
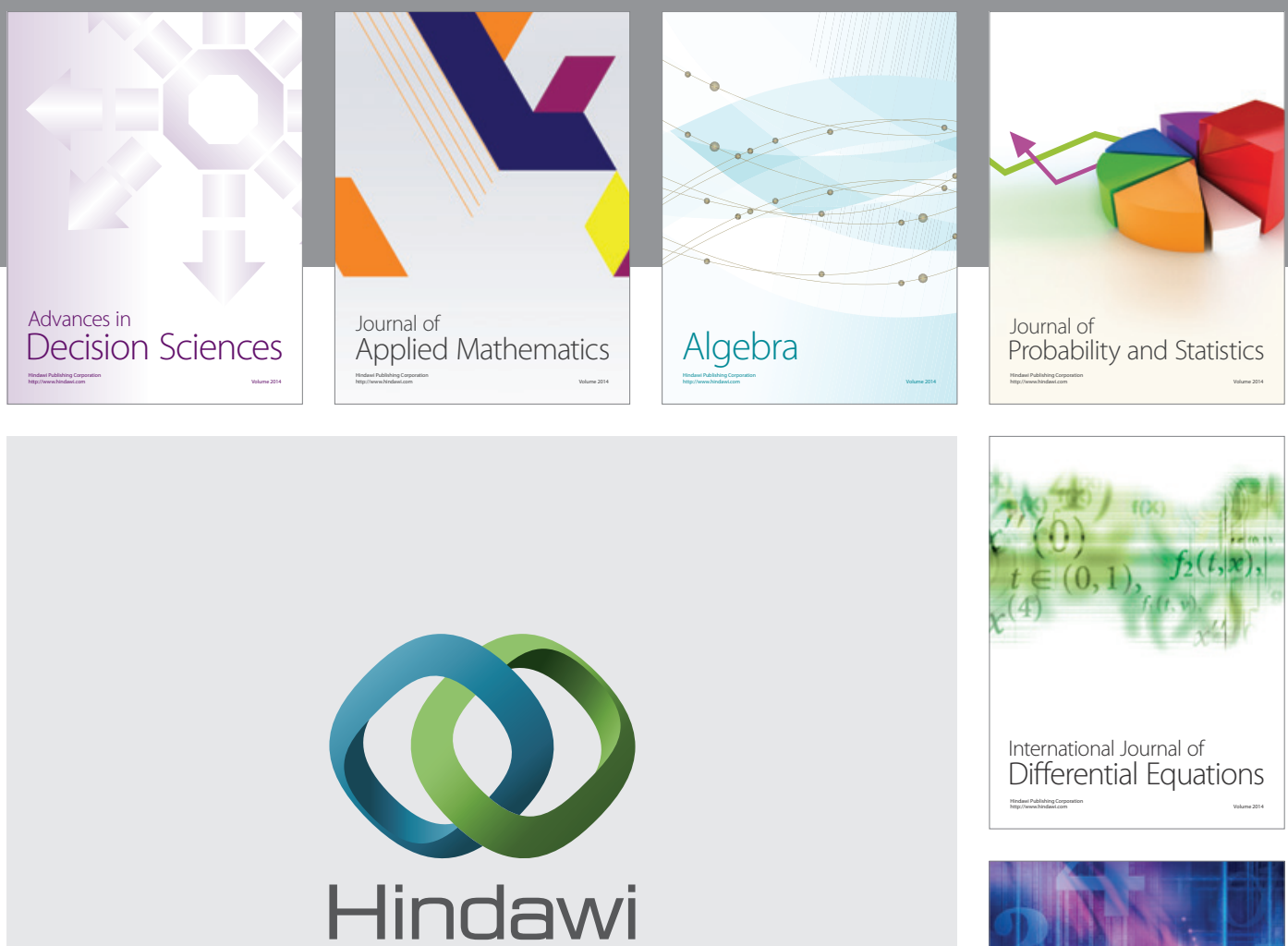

Submit your manuscripts at http://www.hindawi.com
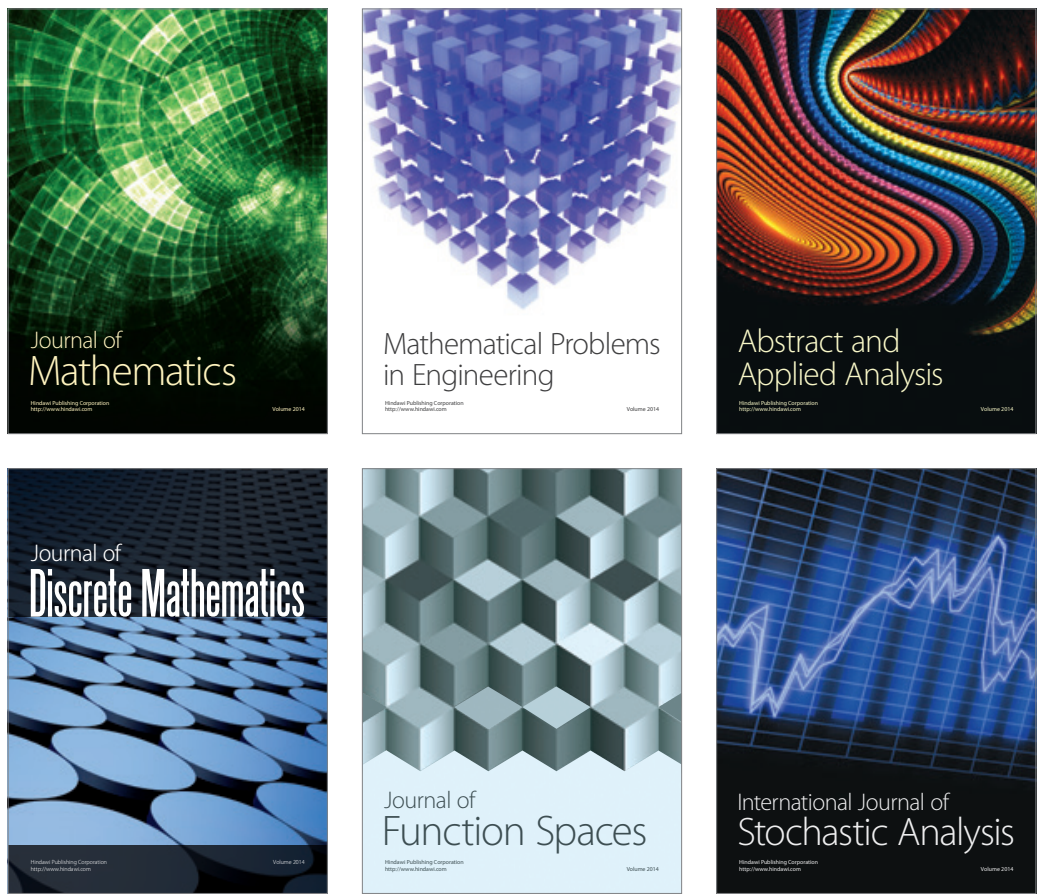

Journal of

Function Spaces

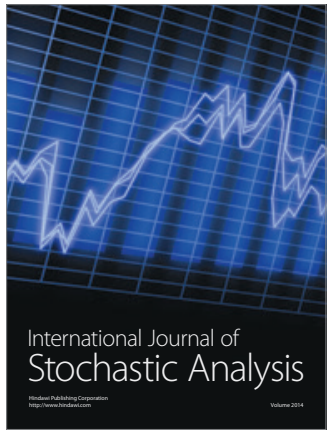

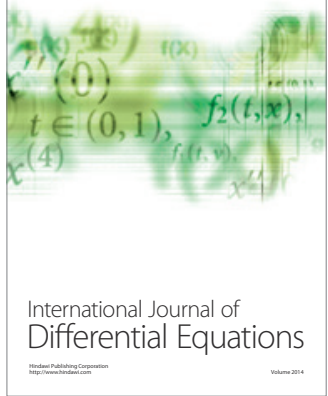
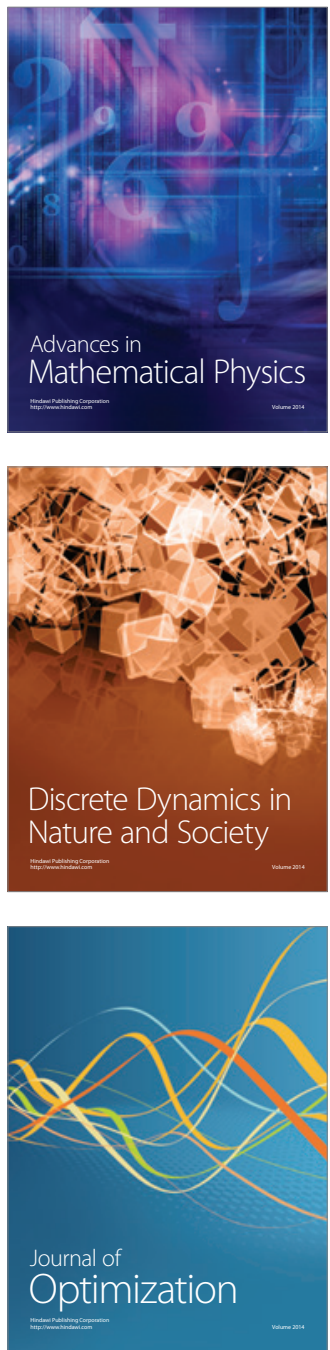